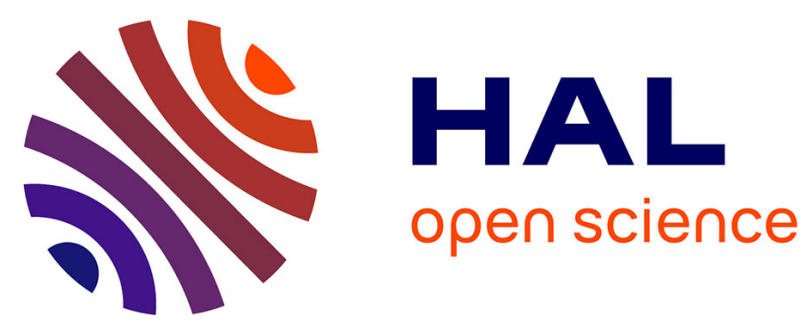

\title{
Techniques de boisages dans les mines d'or gauloises du sud-ouest du Massif central
}

Béatrice Cauuet

\section{To cite this version:}

Béatrice Cauuet. Techniques de boisages dans les mines d'or gauloises du sud-ouest du Massif central. Gallia - Archéologie de la France antique, 2000, Mines et métallurgies en Gaule, 57, pp.129-146. 10.3406/galia.2000.3214 . hal-01902657

\section{HAL Id: hal-01902657 https://hal.science/hal-01902657}

Submitted on 20 Jan 2020

HAL is a multi-disciplinary open access archive for the deposit and dissemination of scientific research documents, whether they are published or not. The documents may come from teaching and research institutions in France or abroad, or from public or private research centers.
L'archive ouverte pluridisciplinaire HAL, est destinée au dépôt et à la diffusion de documents scientifiques de niveau recherche, publiés ou non, émanant des établissements d'enseignement et de recherche français ou étrangers, des laboratoires publics ou privés.

\section{(이) $\$$}

Distributed under a Creative Commons Attribution - NonCommercial - NoDerivatives| 4.0 


\title{
TECHNIQUES DE BOISAGES DANS LES MINES D'OR GAULOISES DU SUD-OUEST DU MASSIF CENTRAL
}

\author{
Béatrice CAUUET
}

Mots-clés. Limousin, mine d'or, dépilage, boisage minier, âge du Fer:

Key-words. Limousin, gold mine, underground work, propping, Iron Age.

Résumé. Les fouilles de plusieurs mines d'or en roche du sud-ouest du Massif central (en Haute-Vienne et en Dordogne) ont permis de retrouver des boisages, datant du second âge du Fer, parfailement conservés en place, dans des dépilages profonds comblés par des sédiments gorgés d'eau. Dans ces chantiers, différentes techniques d'assemblage et de calage ont pu être restituées. L'excellent état de conservation de ces étais facilite l'étude des lechniques de débitage et de façonnage des bois. Leur datation par le radiocarbone el la dendrochronologie situe leur mise en place entre la fin du III s. et la fin du Irr s. avant J.-C. Les différentes dates d'abattage obtenues révèlent des réfections d'étais et l'utilisation de bois de remploi, confirmée par la présence d'aménagements anciens sur certaines pièces. L'ensemble des techniques observées montre une grande maîtrise du travail du bois: un savoir-faire traditionnellement propre aux Gaulois, qui ont le plus souvent préféré la construction à ossature en bois à celle en pierre.

\begin{abstract}
Archaeological excavations of several reef gold mines from south-western Massif central, in Haute-Vienne and Dordogne, have enabled to discover wood propping, tracing back to Second Iron Age, perfectly preserved in situ in deep mining works filled up by watery sediments. In these underground works, different jointing and chocking techniques have been discovered. The excellent state of preservation of these props helps to study the cutting and shaping lechniques of the wood pieces. Radiocarbon data and dendrochronology siluate its setting between the end of the $3^{\text {rd }}$ century and the end of the $1^{\text {rst }}$ century BC. The varied clearing data obtained reveal props repairs and wood pieces reused, confirmed by old workings on some of the props. All the techniques observed show a good mastership of wood working: a traditional ability of the Gaulish people who used wood framework rather than stone.
\end{abstract}

Ces dernières années, la fouille de plusieurs mines d'or en roche en Limousin a révélé de grands travaux d'époque gauloise, exploités à ciel ouvert et en souterrain. Les ouvrages miniers profonds étaient étayés. Dans cette région, le climat humide, avec une pluviosité abondante, et la proximité de la nappe phréatique, atteinte parfois dès $4 \mathrm{~m}$ de profondeur, ont contribué à faire de ces mines des milieux saturés d'eau. Comme les fouilles l'ont montré, les techniques extractives de l'âge du Fer étaient suffisamment abouties pour permettre de travailler sous le niveau de la nappe phréatique en mettant en ouvre des méthodes de drainage, voire de pompage, efficaces (Cauuet, 1991, 1994a, 1995, 1999).

Vers la fin du I ${ }^{\text {er }}$ s. avant J.-C., ces mines ont cessé de fonctionner. L'abandon du drainage a entraîné une remontée naturelle des eaux d'infiltration dans les chantiers profonds, par ailleurs en cours de comblement par les haldes ravinées vers le fond des ouvrages. Le colmatage des travaux profonds, entre 15 et $30 \mathrm{~m}$ de profondeur, par des boues sablo-argileuses perpétuellement gorgées d'eau, a contribué à la conservation in situ des bois des étayages en place dans les ouvrages souterrains. 


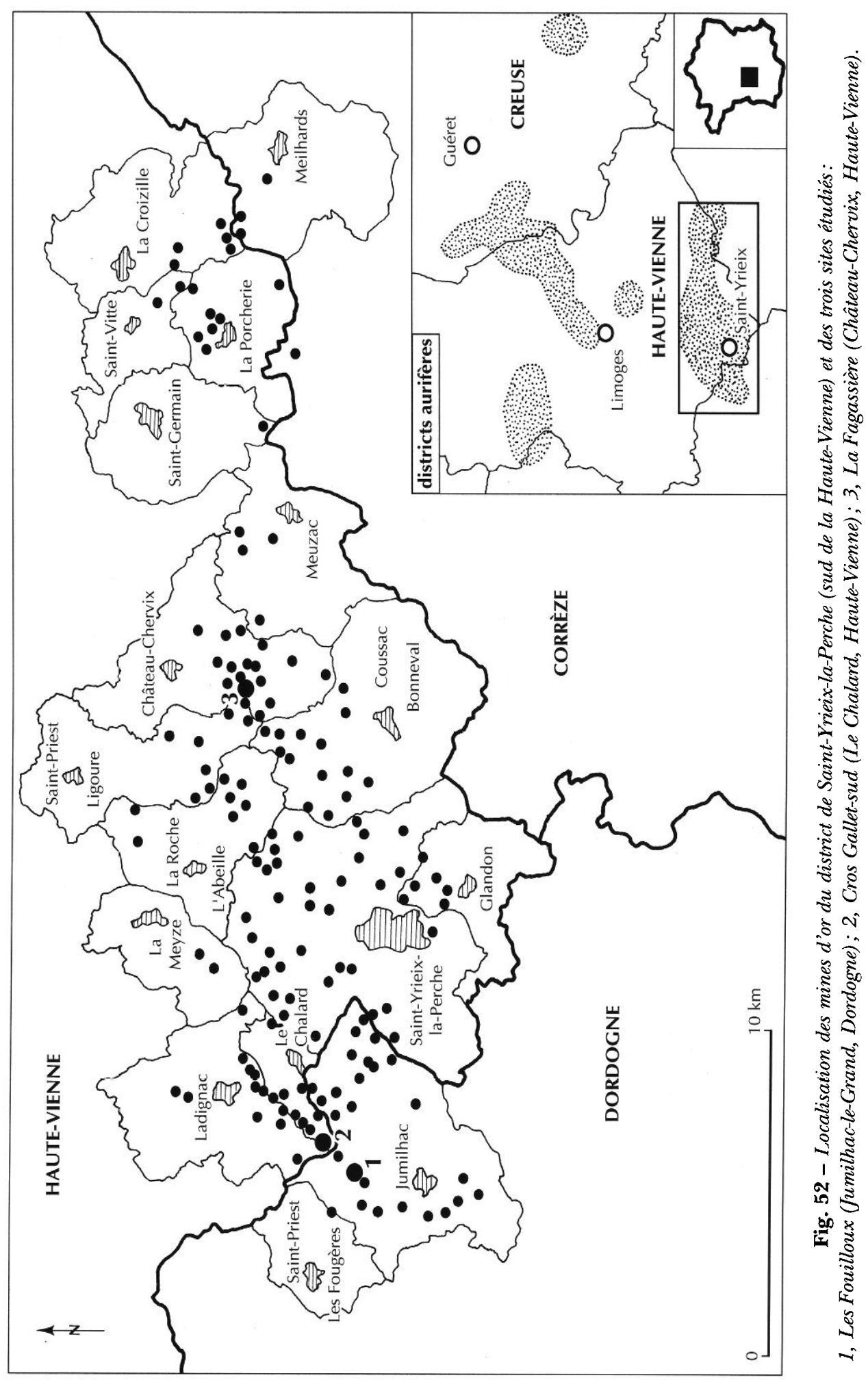


La remarquable conservation des boisages découverts lors des fouilles permet d'en faire une étude complète tant du point de vue chronologique, par l'analyse dendrochronologique, que sous l'angle technologique en s'intéressant aux essences, aux types de débit, de taille et de mise en œuvre des pièces. La mine des Fouilloux (Jumilhac-le-Grand, Dordogne) étudiée de 1988 à 1994, celle de Cros Gallet-sud (Le Chalard, Haute-Vienne) étudiée de 1991 à 1992 et celle de La Fagassière (ChâteauChervix, Haute-Vienne) étudiée de 1995 à 1996, ont livré des lots de boisages remarquables, datables des $\mathrm{II}^{\mathrm{e}}$ et $\mathrm{I}^{\mathrm{er}} \mathrm{s}$. avant J.-C., période de La Tène finale la plus intense pour l'activité minière de la région (fig. 52). Ces boisages constitucnt la matic̀rc de cettc ćtude ${ }^{122}$.

\section{CONDITIONS DES DÉCOUVERTES}

\section{LA MINE DE GROS GALLETSUD}

Dans la mine de Cros Gallet-sud, seuls quelques sondages d'ampleur limitée ont été réalisés (Cauuet, 1991, 1992). L'un de ces sondages, ouvert au fond de l'aurière principale $\mathrm{F} 1$ sur 1,20 à $3,40 \mathrm{~m}$ de largeur et près de $5 \mathrm{~m}$ de longueur, a permis de dégager un dépilage vertical étayé sur toute sa profondeur (fig. 53). Pour des questions de sécurité, la fouille n'a pu être menée au-delà de $4 \mathrm{~m}$ de profondeur à l'intérieur de ce chantier. Elle a dégagé un étayage transversal simple, composé de pièces de bois calées en force, d'un côté, dans des encoches creusées dans la paroi et, de l'autre, bloquées par des planchettes ou des coins de bois (fig. 54).

Le gisement de Cros Gallet a été réexploité de 1982 à 1995, en souterrain tout d'abord, puis à ciel ouvert à partir de 1988. Cette exploitation minière a progressivement fait disparaître les trois aurières majeures, F1, F2 et F3, de cette partie du site, tout en révélant momentanément des travaux gaulois souterrains, comme ce dépilage qui descendait à plus de $8 \mathrm{~m}$ de profondeur ${ }^{123}$. Au moment de

122. Cette étude a bénéficié des observations et des conseils avisés de Philippe Contri, menuisier-ébéniste, qui m’a beaucoup apporté par sa connaissance des bois. Qu'il en soit ici remercié.

123. En 1990-1991, des tensions entre les archéologues et la société exploitante, la SMB (Société des mines du Bourneix, filiale de la COGEMA), n'avaient pas permis d'ouvrir une véritable fouille préventive à Cros Gallet. Seuls quelques sondages ponctuels sur le site en cours de destruction avaient pu être menés.

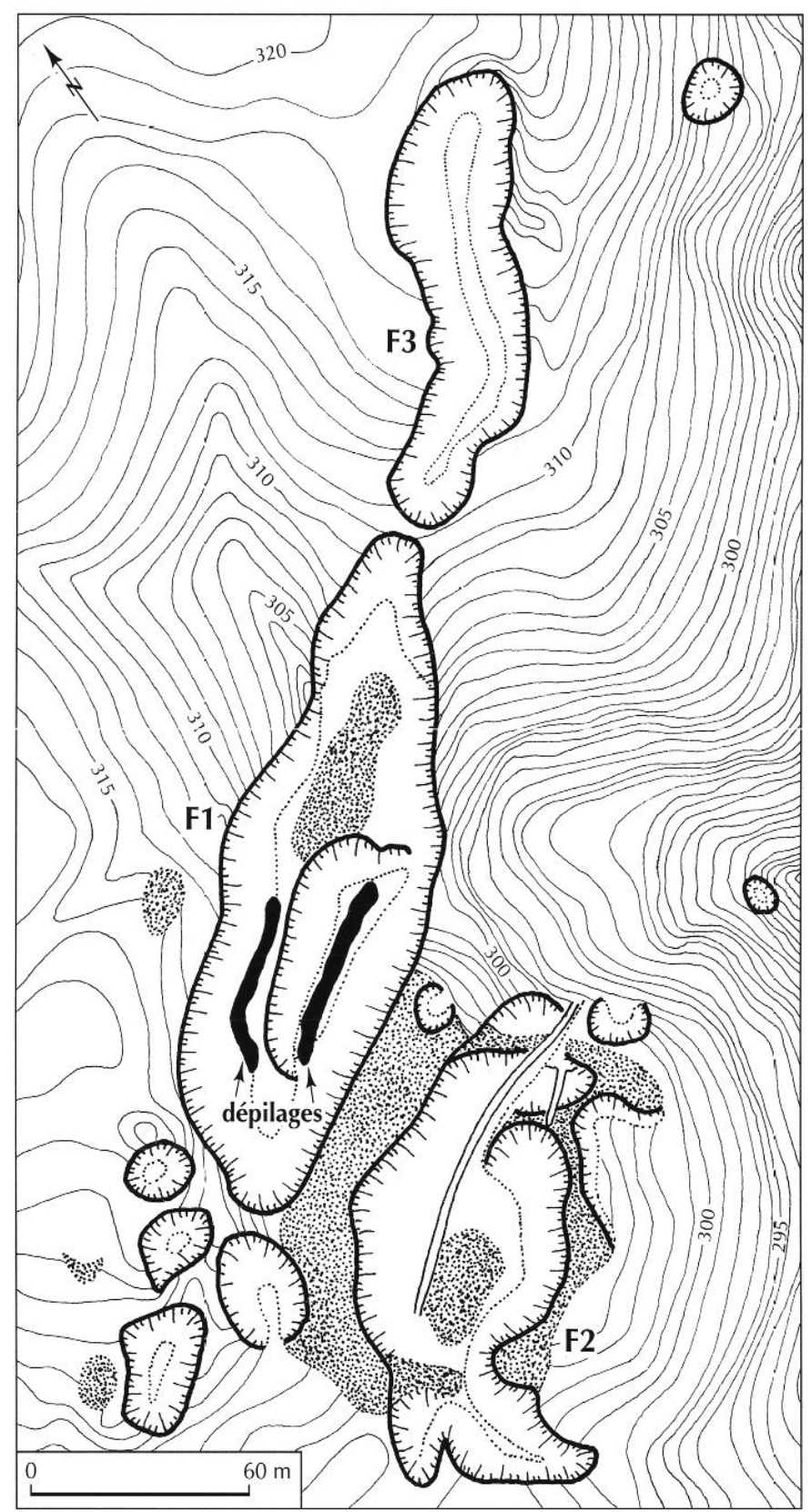

Fig. 53 - Cros Gallet-sud (Le Chalard, Haute-Vienne) : l'aurière F1 aux dépilages étayés.

la fouille, l'exploitation moderne souterraine commencée depuis une dizaine d'années avait entraîné l'assèchement des sédiments enrobant les boisages. De ce fait, les pièces de bois qui n'étaient plus maintenues dans un milieu humide avaient commencé à se décomposer et des pièces entières ou des morceaux d'étais avaient déjà complètement disparu. 


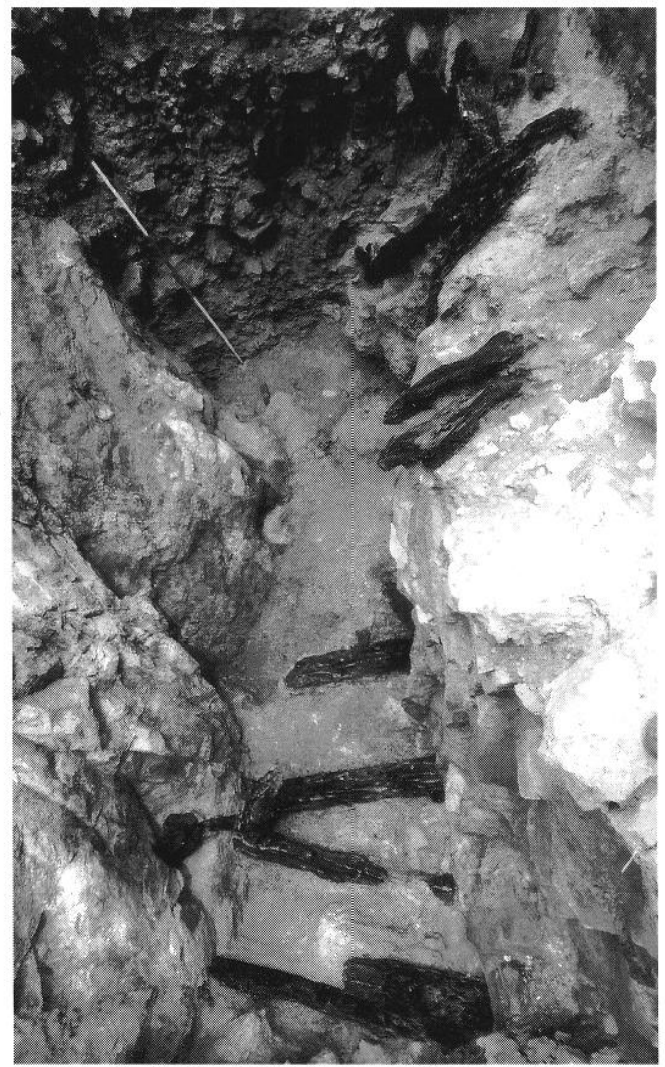

Fig. 54 - Cros Gallet-sud (Le Chalard, Haute-Vienne) : vestiges de boisages encore en place dans le dépilage dégagé dans les profondeurs de la fosse F1.

Les bois prélevés étaient pour l'essentiel du chêne. Il s'agissait de fûts de jeunes chênes d'une section moyenne de $16 \mathrm{~cm}$ (environ 20 ans d'âge), simplement ébranchés et non écorcés (écorce partiellement conservée).

\section{LA MINE DES FOUILLOUX}

À l'inverse de Cros Gallet-sud, la mine des Fouilloux a fait l'objet de fouilles préventives de grande ampleur qui ont permis, notamment, de reconnaître les principaux ouvrages souterrains du site, sinon dans leur totalité, au moins dans des proportions substantielles, suffisantes pour des restitutions de l'ensemble (Cauuet, 1988, 1991, 1992, 1994b). Dans cette mine, des dépilages prolongeaient également le fond des excavations à ciel ouvert (fig. 55). Comme le filon principal du gisement accuse un pendage en moyenne de $40^{\circ}$ vers l'ouest, les dépilages qui ont décaissé ce filon sur 1,50 à $2,50 \mathrm{~m}$ de largeur et près de $8 \mathrm{~m}$ de profondeur sont inclinés (fig. 56 ).

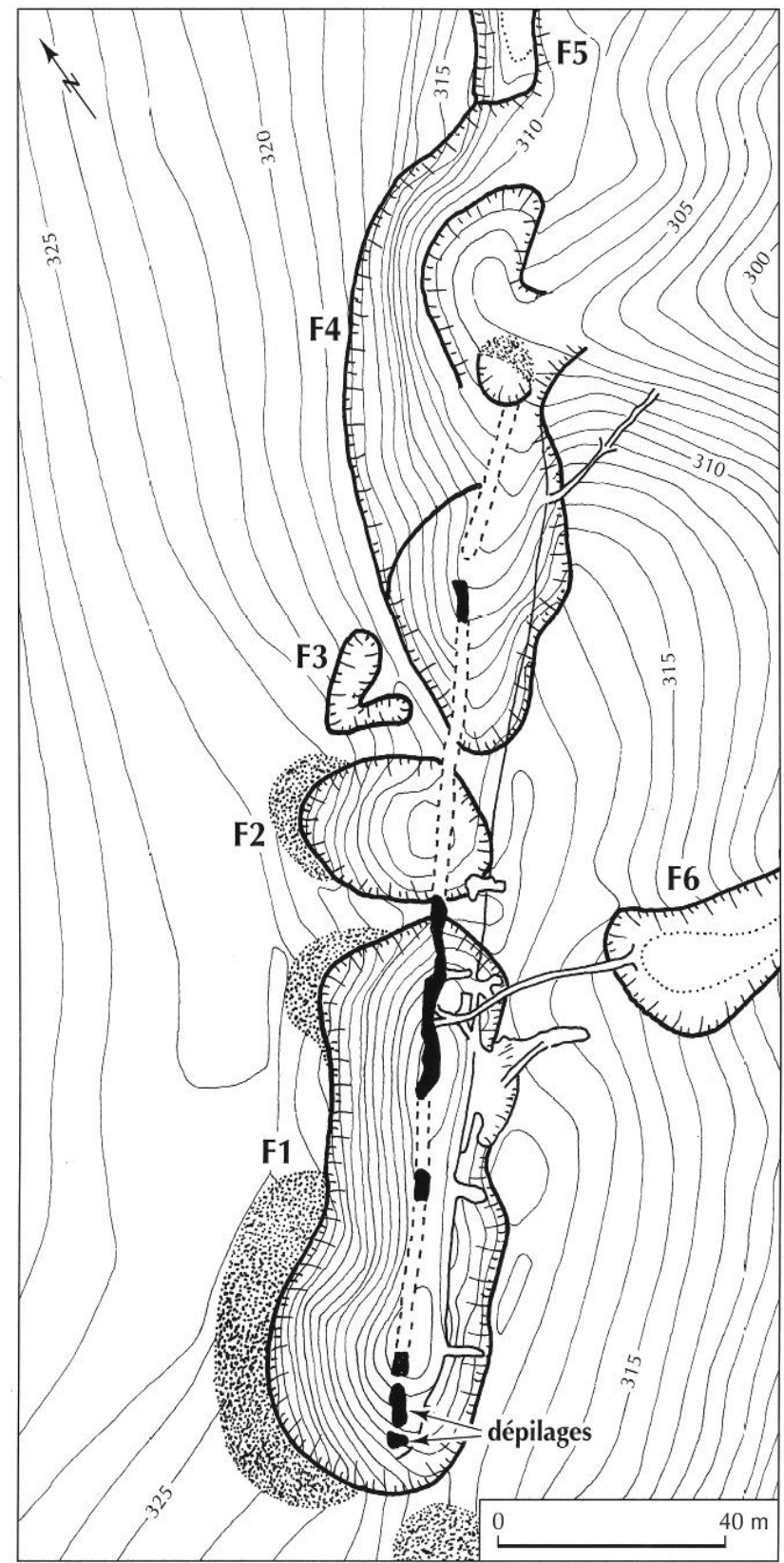

Fig. 55 - Les Fouilloux (Jumilhac-le-Grand, Dordogne) : les cinq aurières ouvertes en enfilade et approfondies par des dépilages étayés.

Compte tenu de la pression des terrains situés au toit du filon, la partie en sous-cavement a dû être fortement étayée sur toute la profondeur des ouvrages. Le site étant resté dans son état d'abandon depuis l'Antiquité, l'ensemble des vestiges étaient encore en place et les boisages ont été retrouvés dans leur logement dans un excellent état de conservation. Le système d'étayage complexe qui caractérisait cette mine a pu être ainsi restitué. 


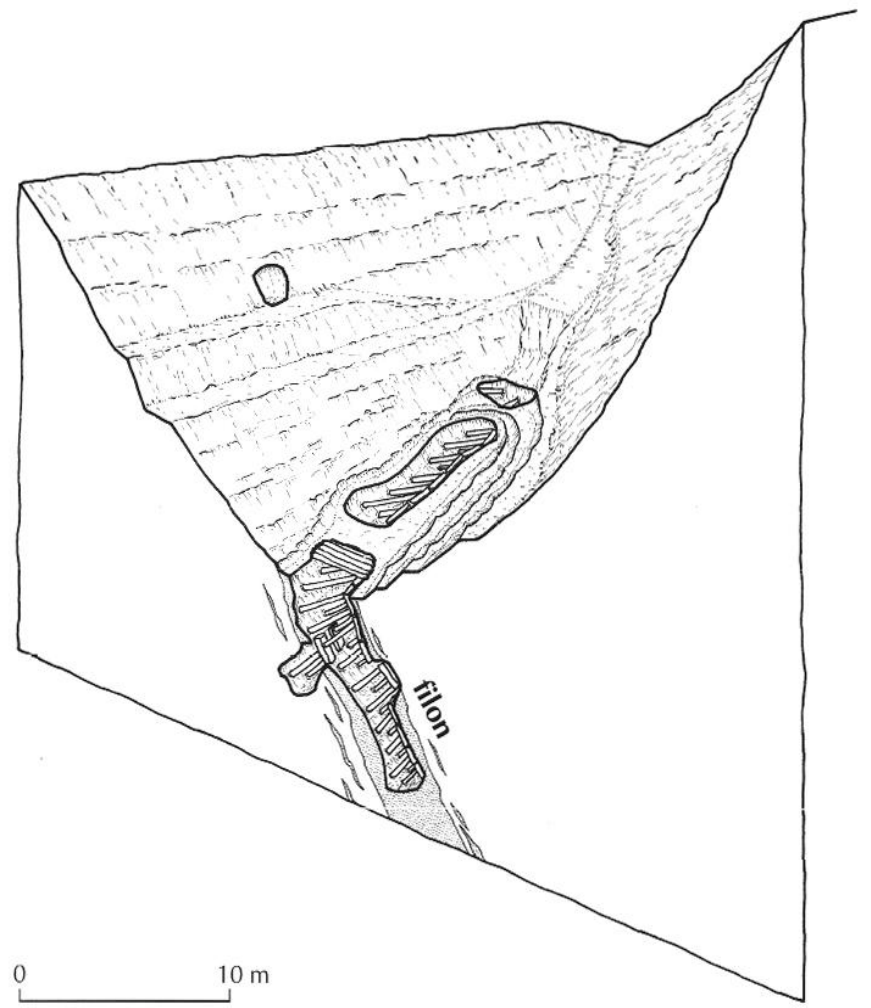

Fig. 56 - Les Fouilloux (Jumilhac-le-Grand, Dordogne) : vue cavalière sur la partie sud de la fosse $F 1$, longs dépilages étayés, foncés en souterrain depuis la mine à ciel ouvert.

La paroi située au toit a été recouverte d'un coffrage de planches, maintenues en place par un étayage dense fait de pièces épaisses, assemblées en " $T$ » ou en « $U$ " par tenons et mortaises. Trois essences ont été observées: chêne et hêtre dominants, quelques pièces en bouleau. Le système de boisage complexe retrouvé aux Fouilloux mettait en œuvre différents types de pièces de boisage. Certaines étaient similaires à celles trouvées à Cros Gallet-sud et correspondaient à des fûts ébranchés, non écorcés et tirés d'arbres jeunes, placés transversalement dans les ouvrages. L'essence dominante pour ces étais peu travaillés était le hêtre, complétée par quelques étais en chêne et en bouleau.

Les pièces montantes assujetties aux étais transversaux et qui plaquaient le coffrage de planches contre la paroi située au toit des ouvrages étaient principalement taillées dans des chênes, souvent de vieux arbres à large tronc et à nombreux cernes. La dureté de ces bois explique que certaines pièces travaillées ont été réutilisées sur le long terme dans les chantiers. En effet, l'ana- lyse dendrochronologique a révélé des écarts chronologiques importants dans les dates d'abattage des arbres entre des ensembles de boisages d'âge homogène et quelques pièces manifestement bien plus anciennes (Cauuet, 1999; Szépertyski, 1999).

Le troisième type de pièces retrouvées, les planches du coffrage, était principalement tiré de gros chênes. Là également, une longue utilisation des planches et des pièces réutilisées a été révélée par l'analyse dendrochronologique.

\section{LA MINE DE LA FAGASSIÈRE}

La mine de La Fagassière a également pu être étudiée de manière approfondie ${ }^{124}$, bien que les conditions de la fouille préventive aient imposé de faire des choix et de limiter nos investigations souterraines à l'étude d'un seul dépilage étayé, DEP3, au fond de la fosse F2 (fig. 57). Dans ce gisement les filons étant subverticaux, les dépilages descendaient verticalement vers les profondeurs. Les étais ont été retrouvés dans ces chantiers dans un excellent état de conservation (Cauuet, 1997a, 1997b, 1999). Le système d'étayage était apparenté au système simple reconnu à Cros Callet-sud. Cependant, l'étude plus complète qui a pu être menée dans le dépilage DEP3 a permis de compléter les informations lacunaires tirées de Cros Gallet-sud.

À la profondeur de $8,50 \mathrm{~m}$ depuis la surface, le fond resserré de la fosse F2, ouverte en gradins, a été approfondi en tranchée descendant verticalement sur plusieurs mètres de profondeur (fig. 58). Ce chantier, large de 1,50 à $2,70 \mathrm{~m}$ et long de $15 \mathrm{~m}$, a été foncé en dépilage souterrain. Il a été dégagé sur $4 \mathrm{~m}$ de profondeur, mais se poursuivait sur plus de $6 \mathrm{~m}$, la fouille ayant dû être stoppée pour des questions de sécurité.

La partie sud du dépilage était équipée d'un boisage simple constitué par des étais transversaux, des fûts de jeunes chênes ou hêtres, ébranchés et non écorcés, calés en force (diamètre moyen 15 à $20 \mathrm{~cm}$ ). Dans la partie nord, à ce système s'ajoutait un dispositif plus complexe

124. Depuis 1993, de bonnes relations prévalent entre la SMB et les archéologues, de sorte que nos interventions sur le terrain, qui bénéficient de la logistique et des moyens mis à notre disposition par la société minière, s'adaptent aux contraintes de l'exploitation, avec pour conséquence des choix à faire dans l'étude des ouvrages qui apparaissent. 


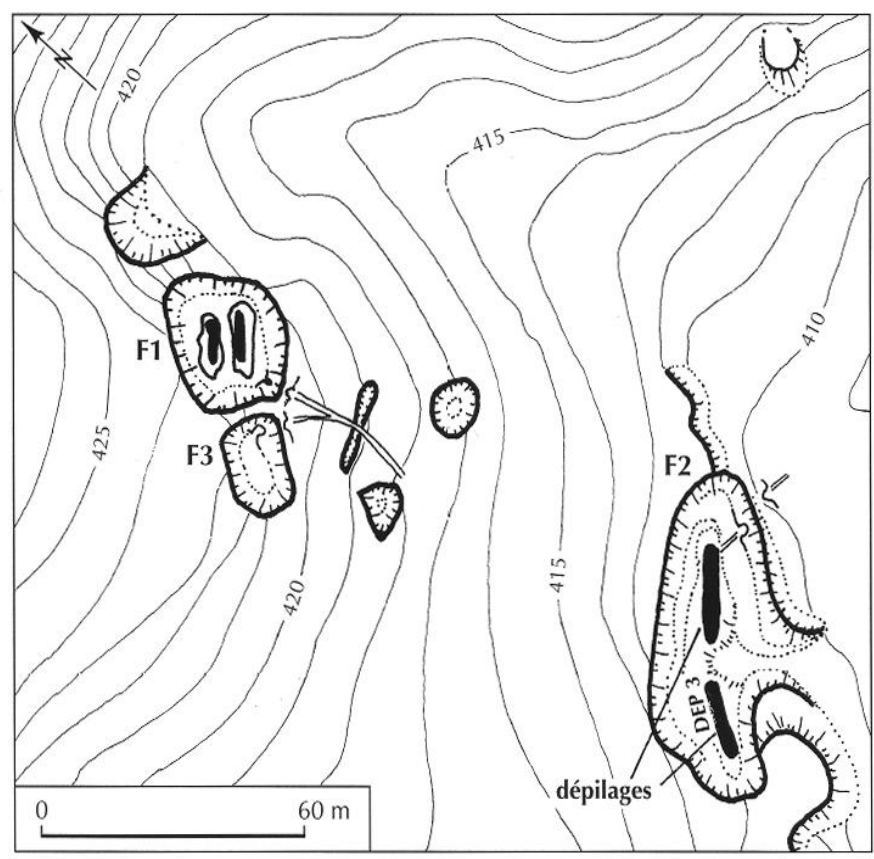

Fig. 57 - La Fagassière (Château-Chervix, Haute-Vienne) : les aurières $F 1$ et $F 2$ aux dépilages élayés.

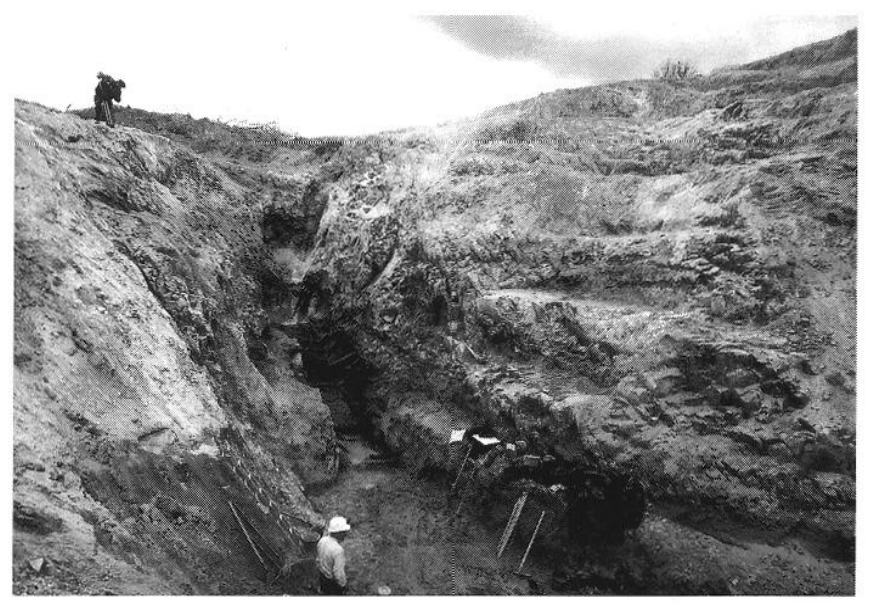

Fig. 58 - La Fugussière (Château-Chervix, Haute-Vienne) : la partie sud de la fosse $F 2$ ouverle en gradins et vue du dépilage étayé en cours de jouille.

permettant de maintenir contre les parois du dépilage un coffrage de planches de chêne par des assemblages d'étais transversaux et latéraux, assujettis en force ou par tenons et mortaises. Dans les différents systèmes observés, les étais sont tenus en force ou par assemblages, aucun clou ou pièce métallique n'est utilisé. Les profondeurs des mines de cette région sont un milieu perpétuellement humide et les bois gonflés par l'eau devaient se maintenir en pression quasi constante dans leur logement.

La fouille de ce dépilage, bien qu'incomplète, a révélé plusieurs aménagements en bois et en remblais qui équipaient le chantier. Il s'agit de plates-formes de travail faites de planches de bois ou d'une murette qui retient des bois et des terres de remblai. Ces platesformes, disposées à divers étages dans le chantier, facilitaient le travail de remontée du minerai et des déblais (Cauuet, 1997a).

\section{APPORTS DE LA DENDROCHRONOLOGIE ET DE LA PALYNOLOGIE}

\section{ANALYSES DENDROCHRONOLOGIQUES}

La bonne conservation des boisages dans les profondeurs des mines du Limousin, avec des parties délicates conservées, comme l'écorce, a permis d'entreprendre des analyses dendrochronologiques dont les apports sont multiples. En premier lieu, les essences sont identifiées et caractérisées. La nature des bois et les conditions de croissance des arbres peuvent être précisées grâce à une étude environnementale et écologique (conditions climatiques, etc.).

Ensuite, la courbe chronologique de référence établie pour le Grand Sud-Ouest de la France par B. Szépertyski (dendrochronologue, responsable du laboratoire d'Analyses et d'Expertises en archéologie et œuvres d'art, LAE de Bordeaux) a permis de calculer à partir de l'étude des cernes de croissance des arbres leur date d'abattage. Une fois cette date connue ou estimée, il est possible de dater précisément la mise en place du boisage dans le chantier (Szépertyski, 1999).

L'âge de l'arbre peut également être établi et révćlcr, par exemple, l'emploi d'arbres jeunes de 15 à $20 \mathrm{~cm}$ de diamètre et d'un âge moyen compris entre 15 et 30 ans. Les arbres jeunes, aux troncs droits, utilisés comme étais transversaux, étaient faciles à tailler et à mettre en place. Le gonflement des bois dû à l'omniprésence de l'eau assurait une excellente tenue des assemblages et des étayages. Dans ce contexte, la date d'abattage peut correspondre à la date de mise en place des étais et aux phases de travail dans la mine. Cependant, la fouille a montré des aires de stockage de bois et des pièces réutilisées portant des aménagements anciens qui indiquent 
une longue utilisation de certaines pièces. Dans ce cas, la date d'abattage des arbres n'est qu'indicative, elle ne permet pas de dater précisément le travail dans le dépilage. Cette réutilisation des boisages a d'ailleurs été confirmée par la datation des bois.

Les analyses dendrochronologiques en cours sur les boisages de la mine des Fouilloux, dont une partie vient d'être publiée (Szépertyski, 1999), ne portent pour le moment que sur le chêne. Les échantillons de bois étudiés ont été synchronisés et ont donné une séquence moyenne de 358 ans. Cette séquence débute en 403 avant J.-C. et se termine en 20 avant J.-C. Un des dépilages a livré des écarts chronologiques importants qui témoignent de bois très anciens, coupés au III ${ }^{e}$ s. avant J.-C. et réutilisés dans le soutènement de chantier en exploitation à la fin du II ${ }^{\mathrm{e}}$ et au début du I ${ }^{\mathrm{er}} \mathrm{s}$. avant J.-C. Un autre dépilage a fait apparaître, d'un côté, des chronologies diverses, ce qui a confirmé les observations faites en fouille et la présence de bois de stockage dans un secteur du chantier abandonné. L'autre partie de ce chantier a livré des chronologies de bois homogènes et la mise en place des boisages soit vers 104 avant J.-C., soit entre 104 et 79 avant J.-C.

Les analyses dendrochronologiques des boisages de Cros Gallet-sud et de La Fagassière sont en cours. Sur ce dernier site, la fouille a livré des planches portant des aménagements anciens, comme de petites mortaises aveugles qui témoignent du remploi de bois. La dendrochronologie pourra sans doute confirmer cette observation.

\section{ANALYSES PALYNOLOGIQUES}

L'étude palynologique d'une mine permet de restituer la vëgètation avant, pendant et après l'exploitation. Pour le moment, les analyses effectuées par M.-F. Diot (palynologue du Centre national de préhistoire de Périgueux) sur les mines du Limousin n'ont porté que sur le site des Fouilloux, intensément exploité de La Tène moyenne à la fin de La Tène finale. L'étude a donné de bons résultats sur les dépilages où les pollens ont été bien conservés dans les sédiments gorgés d'eau.

La composition pollinique a donné $50 \%$ de pollens d'arbres, les autres pollens correspondant à des plantes herbacées de la flore naturelle, aucun pollen de plantes cultivées n'a été décelé. Les pollens d'arbres sont par ordre d'importance: le chêne, l'aulne, le noisetier, le hêtre et le bouleau. En faibles taux, on trouve également le pin, le sapin, le genévrier, le saule, le tilleul et la bourdaine (Diot, 1999). Le diagramme pollinique établi sur le site des Fouilloux est comparable aux types de bois utilisés dans les étayages de la mine. On y retrouve le chêne dominant en accord avec la chênaie diversifiée représentée par les pollens.

Le hêtre et le bouleau sont également présents, mais l'étude des boisages a révélé leur présence en bois d'appoint dans les étayages. L'absence de pollens de plantes cultivées confirme l'éloignement de la mine des habitats permanents des mineurs de La Tène finale, que la fouille et la prospection situent dans un rayon de 500 à $1000 \mathrm{~m}$ autour du site (Cauuet, 1999).

\section{CARACTÉRISTIQUES DES BOISAGES RETROUVÉS}

\section{ESSENCES CHOISIES}

L'étude des bois a permis de reconnaître l'emploi de quatre essences différentes: chêne, hêtre, bouleau pour les éléments de l'étayage et, de manière ponctuelle, le noisetier sous forme de fins rameaux. Devant la diversité des essences utilisées, on peut s'interroger sur le choix de telles essences en s'intéressant aux qualités de ces bois.

Le chêne: la meilleure qualité est représentée par les arbres ayant poussé en forêt. Ils ont un long tronc bien droit et atteignent parfois une hauteur de $30 \mathrm{~m}$ pour un diamètre de $1 \mathrm{~m}$. Le chêne est un bois dur d'une grande durabilité. Il est difficile à travailler en raison de sa dureté, mais résiste bien en milieu humide et est utilisé de ce fait dans la construction navale, les éléments d'écluses et tous les ouvrages extérieurs.

Le hêtre: c'est un bois solide, très dur qui présente un tronc cylindrique. Il est facile à travailler, mais joue beaucoup à l'humidité. Il est très périssable à l'humidité et inadapté aux usages extérieurs (mine?).

Le bouleau: c'est un bois résistant, facile à travailler à la main et qui présente un tronc droit. Cependant, comme le hêtre, il se conserve mal dans des conditions favorisant la pourriture ${ }^{125}$.

125. J.-L. Parmentier, Le grand livre international du bois, Paris, Fernand Nathan, 1980, 276 p. (p. 233, 242 et 254). 
Le noisetier: cet arbre n'est pas utilisé ici pour ses propriétés en tant que bois de fût, mais uniquement pour caler des pièces du boisage au moyen des tiges souples que constituent ses branches jeunes.

Si on avait dû s'en tenir aux propriétés des essences, il apparaît que seul le chêne est un bois aux qualités requises pour le boisage minier. En effet, il allie la solidité et la durabilité en milieu humide. En revanche, le hêtre et le bouleau sont des essences qui résistent bien à la pression, mais sont périssables à l'humidité. Or, en Limousin, la mine profonde est un milieu saturé d'eau.

\section{TYPES DE PIÈCES DE BOIS ET DE MATÉRIAUX}

Les différents systèmes d'étayage rencontrés ont livré quatre types de pièces principales: les étais transversaux ou poussards diagonaux et horizontaux, les madriers ou montants obliques et verticaux, les planches, planchettes et les coins. À ces pièces s'ajoutent des baguettes de bois et des fibres végétales retrouvées en bourrage entre ou derrière les pièces principales. Ces différents éléments peuvent être décrits comme suit:

Les poussards: des étais placés en travers des ouvrages à l'horizontale ou en diagonale; en général de jeunes arbres (entre 15 et 20 ans d'âge) au tronc bien droit (dimensions moyennes: largeur $0,15 \mathrm{~m}$, longueur entre $1,50 \mathrm{~m}$ et $2 \mathrm{~m}$ ). Ils sont en chêne, en hêtre et parfois en bouleau.

Les montants: des pièces larges et épaisses, placées verticalement contre les parois et solidarisées aux poussards. Ils maintiennent généralement un coffrage de planches. Ils sont souvent taillés dans d'anciennes pièces de bois dur, donc en réemploi (dimensions moyennes: largeur $0,20 \mathrm{~m}$, longueur entre 0,70 et $1,20 \mathrm{~m}$ ). Ils sont généralement en bois de chêne.

Les planchettes et les coins: utilisés pour bloquer des poussards ou en calage d'appoint dans un coffrage contre la paroi (dimensions variables). Ils sont essentiellement en chêne, parfois en hêtre.

Les planches: de belles pièces, souvent longues (dimensions moyennes: largeur autour de $0,20 \mathrm{~m}$, longueur variable de 0,60 à plus de $2 \mathrm{~m}$, épaisseur dissymétrique $0,02 \mathrm{~m}$ d'un côté et $0,05 \mathrm{~m}$ de l'autre), généralement utilisées pour l'habillage des parois. Elles sont placées contre la roche, les unes au-dessus des autres, ou posées à l'horizontale sur des poussards et serrées les unes contre les autres pour former des plates-formes de travail (sorte de plancher) à différentes profondeurs des chantiers. Elles sont quasiment toujours en chêne.

Les baguettes: des quarts de rond de branches de noisetier, retrouvés en calage entre les planches formant les plates-formes de travail. La pièce était enfoncée sur l'angle pour jointoyer entre deux planches et les bloquer.

Les paquets de fibres: masses de fibres végétales (utilisation de feuilles et de brindilles à La Fagassière, de fougères et de mousses aux Fouilloux) compactées et mises en garnissage derrière les planches des coffrages. Elles servaient de joint de compression entre le boisage mis en force et les irrégularités des parois. Elles permettaient d'éviter l'écrasement du bois des planches contre la roche. Aujourd'hui dans le boisage des galeries, la Société des mines du Bourneix utilise de la paille.

\section{TRAVAIL DES BOISAGES}

\section{DÉBITAGE DES BOIS}

Une fois les arbres abattus et les branches coupées à la hache (la hachette ou l'herminette), les fûts ont été débités principalement par refente des grumes. La refente est une technique de réduction du bois d'œuvre rond en plateaux ou en planches, sans utilisation d'une scie, afin de préserver l'intégrité des fibres. Pour de nombreuses essences, comme le chêne, un artisan expérimenté peut fendre une grume de bout en bout en entaillant - à la hache ou avec un coin en fer - l'extrémité du fil exactement selon l'un des rayons. Ces derniers sont les lignes des cellules de réserve disposées radialement à partir du centre du tronc.

Les deux demi-troncs sont ensuite refendus en billettes de section triangulaire ou en planches plates, toujours au moyen des mêmes outils. Cette méthode étant rapide, facile et nécessitant des outils simples, les pièces, comme des planches de refente, peuvent être fabriquées immédiatement lors de défrichements. De la même manière, les tiges fines de certains arbres jeunes, comme le noisetier, peuvent être fendues ou simplement dédoublées avec un couteau ou une serpe ${ }^{126}$. Le débit des pièces étudiées se caractérise ainsi:

126. Voir note 125: J-L. Parmentier, op. cil., 1980, p. 148. 


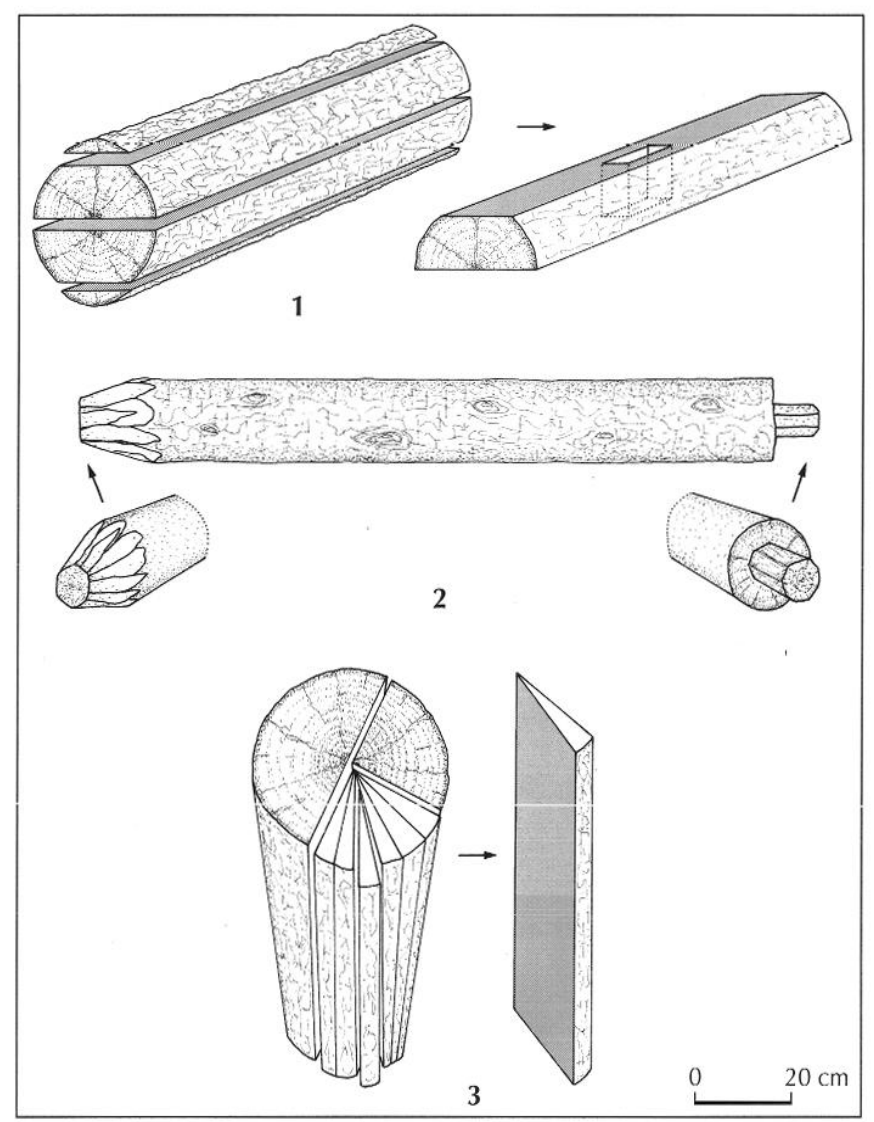

Fig. 59 - Débil et travail des bois utilisés dans les dépilages gaulois: 1, grume débitée sur dosses donnant deux plateaux, un des plateaux percé d'une mortaise centrale débouchante; 2 , tronc ébranché aux extrémités taillées en tronc de cône el en tenon cylindrique; 3, grume débitée en rayons, un débit radial donnant 24 planches de refente de section conique.

Les poussards: il s'agit de troncs ébranchés et d'arbres jeunes aux fûts bien droits (fig. $59, \mathrm{n}^{\circ} 2$ ). Ces pièces retrouvées avec l'écorce conservée sont excellentes pour l'analyse dendrochronologique et la précision de la date d'abattage. Malheureusement les pièces en chêne, seule essence datable pour le moment, sont rares.

Les montants: ils correspondent aux grosses pièces mortaisées; ce sont des grumes débitées sur dosses (fig. 59, $\mathrm{n}^{\circ}$ 1). Dans un même fût, on tire deux montants et on perd deux dosses (parties du dessus enlevées). Travail fait aux coins de fer et à la masse en fer ${ }^{127}$. Sur les plateaux débités une partie de l'écorce est encore

127. J. IIeurtematte, R. Keller, Le sciage du bois, Poiticrs, ćd. dc la Librairie Delagrave, 1979, 144 p., 541 fig. (p. 82-83). présente. Ces pièces, généralement en chêne, ont de nombreux cernes et sont également excellentes pour la dendrochronologie.

Les planches, planchettes et coins en bois: à partir d'un fût débité en quartiers, débit des planches en rayons, quartier par quartier (fig. 59, $\mathrm{n}^{\circ} 3$ ). Ce travail fait aux coins de fer et à la masse en fer permet d'obtenir de longues planches, de section triangulaire caractéristique. Les planchettes et les coins en bois, aux petites dimensions très variables, correspondent à des chutes de planches récupérées et recoupées (scie ou herminette?). Les planches en chêne sont tirées de larges fûts et sont aussi excellentes pour la dendrochronologie.

Les baguettes: jeunes branches de noisetier, bien droites, coupées en quartiers (travail à la hachette, à l'herminette ou à la serpe).

Les masses de fibres: à La Fagassière, une partie des chutes de branches, le feuillage terminal, a servi de garnissage derrière les planches des coffrages. Les dosses et les branches intermédiaires devaient alimenter les feux entretenus dans la mine pour l'abattage de la roche au feu et aux abords pour le grillage du minerai en cours de traitement. Ainsi aucune partie des bois ne devait se perdre.

\section{TAILLES DES PIÈCES}

Les étayages complexes à assemblages à tenons et mortaises impliquaient de travailler les pièces utilisées.

Les poussards: seules les extrémités étaient travaillées (fig. 59, $\mathrm{n}^{\circ} 2$ ); en taille tronconique faite à l'herminette (traces de l'outil encore bien visibles), un travail rapide qui reprenait à peine la forme donnée au départ par l'abattage de l'arbre à la hache ou à l'herminette; en taille droite (sans doute à la scie, mais traces peu visibles?) ; en taille en tenon de forme grossièrement cylindrique ( $7 \mathrm{~cm}$ de diamètre en moyenne), un travail fait à l'herminette ou au ciseau à bois en fer frappé par une massette en bois. L'assemblage à tenons et mortaises étudié ici se faisait entre un tenon cylindrique et une mortaise quadrangulaire. Cela laissait un peu de jeu entre les pièces et facilitait leur emboîtement en force.

Les montants: leurs extrémités étaient sciées droit ou grossièrement taillées à l'herminette (coups d'outils 
Fig. 60 - Boisage des Fouilloux (Jumilhac-le-Grand, Dordogne) : traces de coups d'herminette visibles sur une face taillée d'un montant.

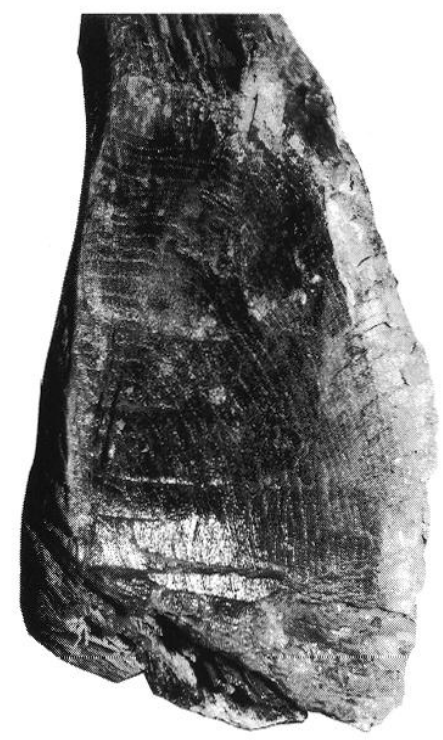

visibles, largeur de l'outil mesurable). Ces montants étaient ensuite percés d'une ou deux (voire trois) mortaises, selon la longueur de la pièce. La troisième mortaise n'a pas pu être observée sur pièce, car les montants trop longs ont été retrouvés brisés. Ces mortaises, de section quadrangulaire (environ $10 \mathrm{~cm} \times 10 \mathrm{~cm}$ ), étaient débouchantes (ouvertes au travers de la pièce) et creusées au ciseau à bois en fer (traces de l'outil visibles et mesurables) frappé par une massette en bois (fig. 59, $\left.n^{\circ} 1\right)$.

Les planches, planchettes, coins et baguettes: ces pièces n'étaient pas travaillées et étaient utilisées brutes de débit, seulement recoupées dans la longueur ou la largeur (à la scie? ou à l'herminette), en fonction des besoins de l'étayage (fig. $59, n^{\circ} 3$ ).

\section{TRACES ET TYPES D'OUTILS}

Bien que les fouilles n'aient pas encore livré d'outils de mineurs, et notamment de boiseurs, les techniques de débitage et les traces d'outils laissées dans le bois permettent de restituer le type de travail effectué et l'outillage employé. Sur certaines pièces des traces de coups d'herminette, largeur: $6 \mathrm{~cm}$ (fig. 60 ) et de coups de ciseau à bois, largeur: $2,5 \mathrm{~cm}$ (fig. 61) sont encore bien visibles et conservées dans le bois.

L'utilisation d'une scie ou d'une hache est déduite (ou supposée) en fonction du type de taille ou de coupe observé, mais elle n'est pas encore attestée par des traces

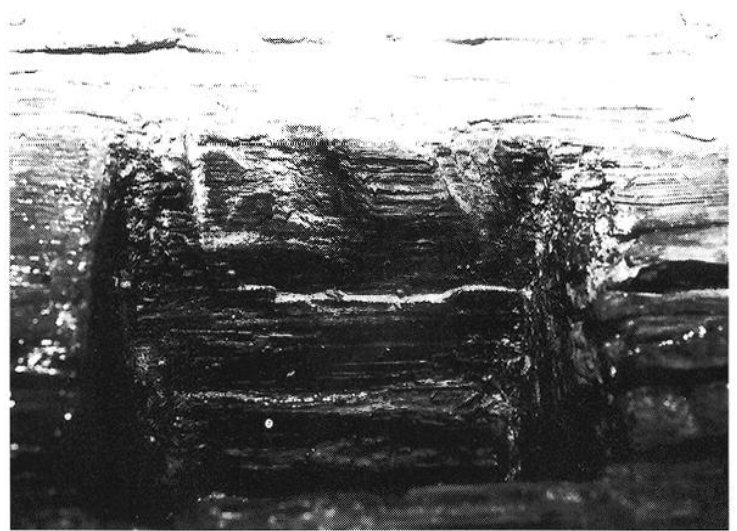

Fig. 61 - Boisage des Fouilloux (Jumilhac-le-Crand, Dordogne): traces de coups de ciseau à bois visibles sur le bord d'une mortaise.

identifiables. Beaucoup de lots de boisages restent à étudier, d'autres types de traces pourront être encore observées. L'outillage en fer du boiseur devait se résumer à des coins, une massette, une hache ou hachette, une herminette et des ciseaux à bois. La scie a pu être employée ponctuellement, par exemple, pour obtenir des coupes droites des étais transversaux ou des tenons. Une massette en bois et des coins en bois dur pouvaient compléter l'équipement.

\section{LES SYSTÈMES D'ÉTAYAGE}

Le pendage du chantier a conditionné le choix du système d'étayage. Dans un chantier aux parois subverticales, on pouvait mettre en place un étayage simple et/ou complexe, selon la résistance locale des parois de l'ouvrage. Les deux systèmes pouvaient cohabiter. Dans un chantier incliné accusant un pendage de près de $50^{\circ}$, seul un étayage complexe était installé.

\section{ÉTAYAGE SIMPLE}

Il consiste à placer en force des séries d'étais, des troncs ébranchés de jeunes arbres (20 ans d'âge environ), disposés en travers de l'ouvrage. Les extrémités des pièces de bois sont taillées différemment en fonction du type de calage adopté. Deux types de calage ont été observés. 
Un calage en force contre la paroi de l'ouvrage:

- extrémité de l'étai coupée droit, vraisemblablement à la scie mais sans trace évidente encore observable sur la pièce;

- une ou plusieurs planchettes, souvent utilisées là en remploi, ou des coins longs (longueur $20 \mathrm{~cm}$, largeur 8 à $10 \mathrm{~cm}$ ) sont intercalés entre le bout de l'étai et la paroi (fig. 62).

Un calage de l'étai dans une encoche creusée dans la paroi. L'extrémité de l'étai a été taillée à l'herminette en tronc de cône (traces de coups d'outil visibles dans le bois). L'encoche a été creusée par une série de coups de pointerolle portés en éventail et encore bien marqués dans la roche.

\section{ÉTAYAGE COMPLEXE}

Des étais transversaux maintiennent un coffrage de planches contre la paroi, soit par un calage en force avec une pièce montante, soit par un assemblage à tenons et mortaises entre un étai transversal tenonné et une épaisse pièce montante mortaisée. Ces deux systèmes de blocage des pièces peuvent se rencontrer dans un même ouvrage ouvert en tranchée. Dans un chantier creusé obliquement, seul l'assemblage de pièces à tenons et mortaises a été observé.

\section{ÉTAYAGE DE CHANTIER INCLINÉ}

Dans ce type de chantier ( $1,20 \mathrm{~m}$ à $2 \mathrm{~m}$ de large), le haut de l'ouvrage correspond au toit du filon, c'est la partie vulnérable qui doit être soutenue. Le bas de l'ouvrage est situé au mur du filon (partie inférieure) et dans un terrain stable qui ne bougera pas. Il n'y a donc pas lieu de mettre un coffrage sur cette paroi qui représente la sole du chantier. En revanche, elle sert de base d'appui pour l'ensemble du boisage.

À la mine des Fouilloux, les ouvrages profonds désobstrués, entre les cotes -20 et $-30 \mathrm{~m}$, présentaient tous un profil incliné selon un pendage vers l'ouest de 45 à $50^{\circ}$. Cette inclinaison suivait le pendage du filon exploité. Dans cette mine, les étayages observés étaient à $90 \%$ constitués de pièces de bois assemblées par tenons et mortaises. Elles servaient à maintenir la paroi surplombante et à bloquer un coffrage de planches qui couvraient le toit de l'ouvrage. Les $10 \%$ des étais

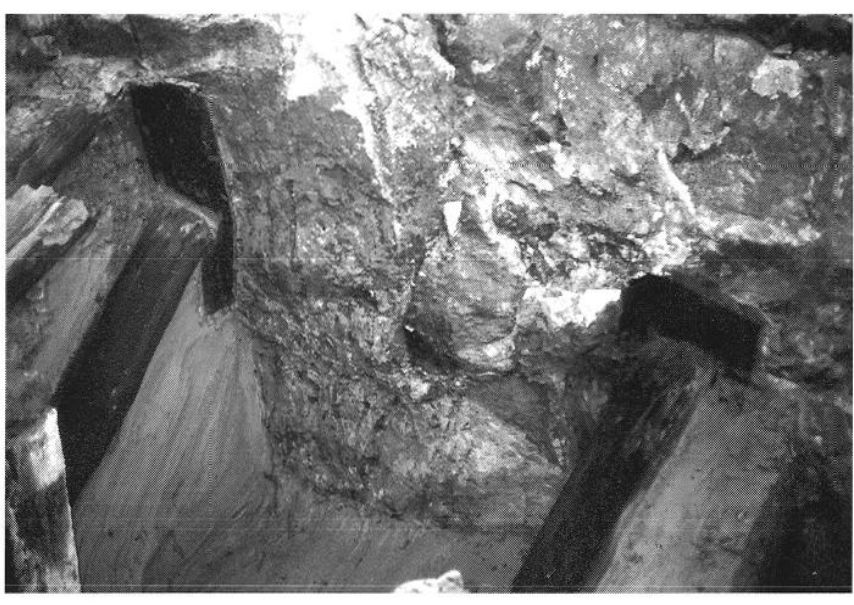

Fig. 62 - La Fagassière (Château-Chervix, Haule-Vienne) : étayage simple avec des planchetles ulilisées pour caler des étais transversaux.

restants correspondaient à un étayage simple fait de pièces calées en force par des planchettes ou dans des encoches et mis en place dans de petits dépilages aux parois verticales.

Dans les dépilages inclinés, l'étai placé en travers de l'ouvrage, appelé poussard diagonal, était calé dans une encoche creusée dans la paroi de la sole (côté mur du filon). L'extrémité était taillée en tronc de cône pour bien s'emboiter dans l'encoche (fig. 59, $\mathrm{n}^{\circ} 2$ ). Ce type d'étai, un tronc ébranché d'arbre jeune, était le plus souvent en hêtre, parfois en chêne ou en bouleau (entre 10 et $20 \%$ des pièces). L'abattage d'arbres jeunes fournissait des fûts bien droits, au diamètre compris entre $14 \mathrm{~cm}$ et $18 \mathrm{~cm}$. L'autre extrémité de l'étai était taillée en tenon grossièrement cylindrique, vraisemblablement à l'herminette et à la scie (?).

Le tenon du poussard diagonal était emboîté dans une mortaise débouchante de section grossièrement carrée, percée au travers d'un plateau de bois. Cette pièce, une grume débitée sur dosse (fig. 59, $\mathrm{n}^{\circ} 1$ ), avait des dimensions variables: longueur entre 0,80 et $1,50 \mathrm{~m}$, largeur entre $0,22 \mathrm{~m}$ et $0,30 \mathrm{~m}$, épaisseur entre $0,10 \mathrm{~m}$ et $0,18 \mathrm{~m}$. Les pièces courtes (environ $0,80 \mathrm{~m}$ de long) étaient percées d'une mortaise centrale. Un montant court constituait avec un poussard tenonné un assemblage en « $T$ ». Les pièces longues ( $1,40 \mathrm{~m}$ et plus de longueur) étaient percées de deux mortaises placées vers chaque extrémité. Un montant long était donc assujetti à deux poussards tenonnés en constituant aussi un assemblage en « $U$ " (fig. 63). 


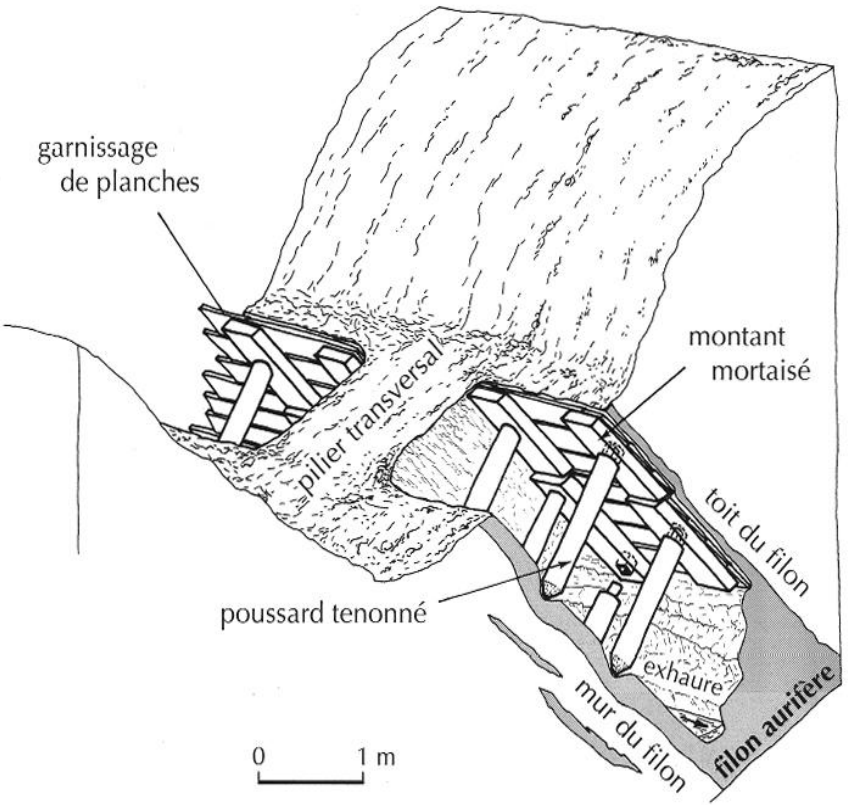

Fig. 63 - Les Fouilloux (Jumilhac-le-Grand, Dordogne): vue cavalière des dépilages inclinés et de leur élayage complexe.

Sur un plan horizontal, on plaçait des poussards tous les $0,50 \mathrm{~m}$ environ (écart d'entre axes $0,85 \mathrm{~m}$ ) et sur un plan vertical, on retrouvait ces pièces à $1 \mathrm{~m}$ d'intervalle les unes des autres. L'emploi de montants courts ou longs se rencontrait indifféremment dans le boisage, l'écartement entre poussards restant régulier. Les montants mortaisés, placés verticalement (selon le pendage du chantier), plaquaient en force un coffrage fait de planches débitées en rayons (fig. $59, \mathrm{n}^{\circ} 3$ ) et disposées les unes au-dessous des autres contre la paroi du toit de l'ouvrage.

Les montants mortaisés et les planches sont en chêne pour la plupart. Certaines datations dendrochronologiques obtenues sur ces pièces ont montré des écarts d'âges trop importants avec ceux des poussards. Il faut en déduire un remploi de certaines pièces au cours du temps. Planches et montants, tirés souvent de très larges fûts de chênes centenaires (Szépertyski, 1999), semblent avoir constitué des pièces de bois précieuses que l'on aura fait durer le plus longtemps possible. Il en résulte que la seule datation (date d'abattage) de ces pièces peut être sujette à caution pour le raisonnement sur la chronologie d'ensemble d'un chantier, car la date d'abattage de certaines pièces de chêne peut remonter à plus d'un siècle avant la date finale de mise en place du boisage.

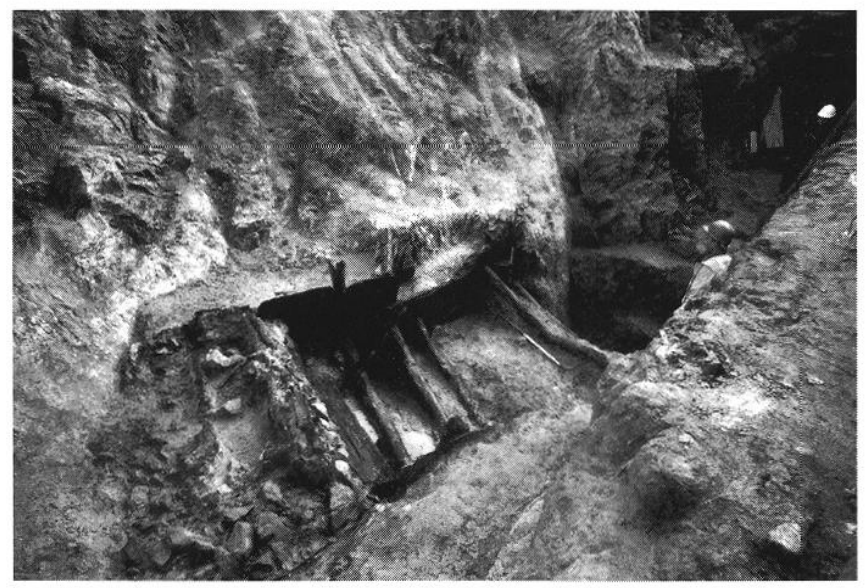

Fig. 64 - La Fagassière (Château-Chervix, Haute-Vienne) : vue d'ensemble sur la partie nord du dépilage fouillé côté sud de la fosse $F 2$, et occupé par un étayage complexe et une plate-forme bâtie. en remblais, murette el bois.

\section{ÉTAYAGE DE CHANTIER VERTICAL}

Ce type d'ouvrage a le profil d'une tranchée large de 2 à $2,50 \mathrm{~m}$, creusée sur 8 à $10 \mathrm{~m}$ de profondeur. Comme dans les chantiers inclinés, les boisages sont mis en place, étage après étage, de haut en bas et au fur et à mesure de l'approfondisscment de l'ouvrage. Pour atteindre les parties profondes, les boiseurs devaient donc se passer les nouvelles pièces de bois préparées à travers une « forêt » d'étais.

Les mines de Cros Gallet-sud et de La Fagassière ont livré des dépilages verticaux, étayés selon des dispositifs similaires. L'étude plus complète menée en 1996 à La Fagassière a révélé l'emploi d'étayage simple et complexe dans un même chantier, tandis qu'à Cros Galletsud l'emploi seulement d'un étayage simple. L'étayage complexe avait été réservé à une partie de l'ouvrage aux parois faillées et très instables (fig. 64).

En chantier ouvert en tranchée, les risques d'effondrement viennent des parois verticales. Il fallait donc coffrer les parois opposées en maintenant un coffrage par des étais horizontaux placés transversalement et assujettis à des pièces montantes, soit en force, soit par assemblage à tenons et mortaises. À La Fagassière, nous avons trouvé dans le même secteur d'un chantier ces deux systèmes de calage.

Dans les assemblages mis en force, deux à trois étais transversaux pouvaient maintenir un même montant à différentes hauteurs. Cette pièce, une planche longue et 


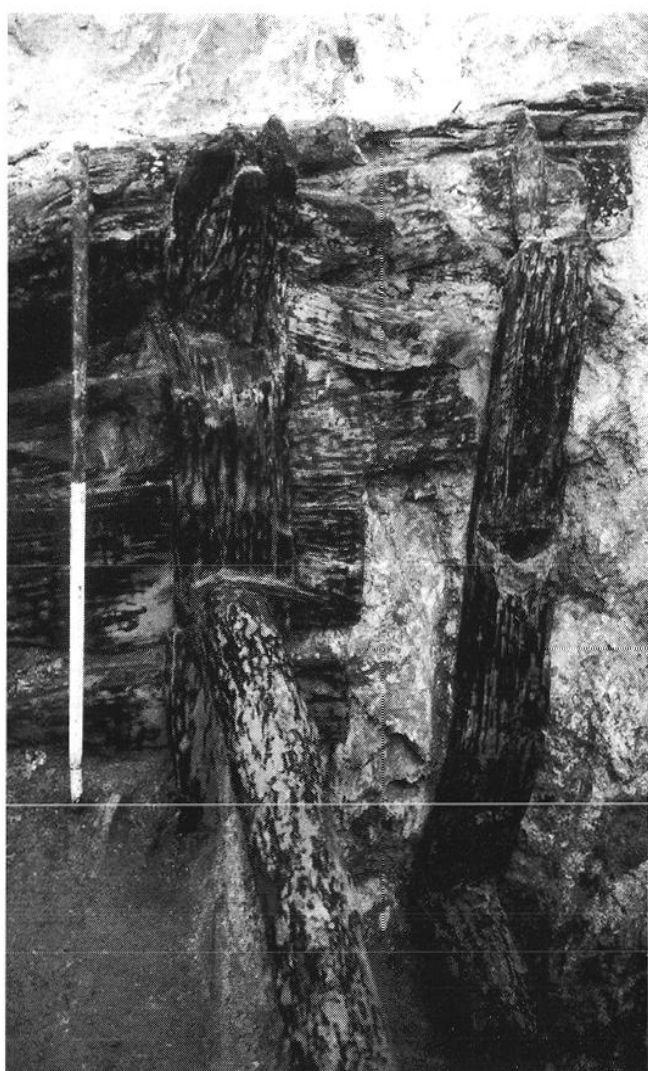

Fig. 65 - La Fagassière (Château-Chervix, Haute-Vienne) : coffrage de planches tenu contre la paroi par des montants bloqués par des étais transversaux coupés droit.

épaisse, était plaquée verticalement contre les planches du coffrage, elles-mêmes disposées horizontalement contre la paroi les unes au-dessous des autres. Dans ce cas, les deux extrémités des étais étaient coupées droit (à la scie?), des planchettes et des petits coins servant parfois à les bloquer contre le montant (fig. 65).

Enfin, un dispositif plus compliqué encore que celui observé aux Fouilloux a été retrouvé à La Fagassière. Dans le haut et à l'extrémité nord d'un des dépilages, une sorte de caisson avait été mis en place. Des étais transversaux placés à l'horizontale étaient tenonnés aux deux extrémités. Ils étaient emboîtés par deux (voire par trois) à un même montant de chaque côté du chantier. Les montants (longueur: $1,50 \mathrm{~m}$ à $2 \mathrm{~m}$, largeur: $0,25 \mathrm{~m}$, hauteur: $0,15 \mathrm{~m}$ ) étaient percés aux extrémités (voire aussi au milieu) de deux (ou trois) mortaises débouchantes, de section carrée. Ils étaient eux-mêmes adossés à un coffrage de planches qui couvrait les parois. Un tel assemblage de quatre ou cinq étais, par tenons et mortaises en forme d'échelle, devait être mis en place à l'extérieur du chantier, puis glissé et calé en force dans la tranchée contre les planches du coffrage. Ce dispositif très complexe n'a été retrouvé qu'en deux exemplaires. Ces deux « échelles » délimitaient un espace quadrangulaire de $2,40 \mathrm{~m}$ de côté, fortement consolidé dans le dépilage (Cauuet, 1999, p. 50).

\section{PLATES-FORMES DE TRAVAIL}

L'organisation de l'espace minier à l'intérieur des dépilages associait au système d'étayage en bois l'aménagement de plates-formes de travail (fig. 66 ct 67). Certaines de ces plates-formes étaient constituées par des planchers comprenant d'épaisses planches de chêne, des demis et des quarts de troncs de chêne ou de hêtre. maintenus serrés les uns contre les autres et en appui sur les étais transversaux du boisage. Quelques baguettes de bois faites de quarts de ronds, tirés de jeunes branches de noisetier, venaient jointoyer en force cette disposition des pièces, ainsi bloquées sans utilisation de clous. Un autre type d'aménagement a été construit avec des déblais miniers, armés de pièces de bois et maintenus par un coffrage de planches et des blocs de quartz.

\section{PLANCHERS EN BOIS}

À la mine des Fouilloux, les vestiges d'une plate-forme en plancher ont été dégagés à la partie supérieure et à l'extrémité sud d'un des dépilages (Cauuet, 1994b, p. 115-119; 1999, p. 47-53). Mais, c'est la mine de La Fagassière qui a livré les plus beaux exemples de ces aménagements.

À La Fagassière, le dépilage DEP3, ouvert côté sud de la fosse F2 (fig. 57), a été fouillé sur près de $5 \mathrm{~m}$ de profondeur ${ }^{128}$. Deux plates-formes constituées par des planchers de bois ont été découvertes, conservées en place dans le chantier. La plate-forme supérieure $(1,70 \mathrm{~m} \times 2,20 \mathrm{~m})$ avait été aménagée à l'extrémité sudouest, posée sur les premiers étais du boisage (Cauuet, 1997a, p. 203-204). Elle prolongeait sur environ $3,80 \mathrm{~m}^{2}$

128. Cette mine a été étudiée dans le cadre d'une fouille préventive en 1996-1997. Les contraintes de temps et de sécurité n'ont pas permis d'atteindre le fond des ouvrages dans cette partie de la mine. 


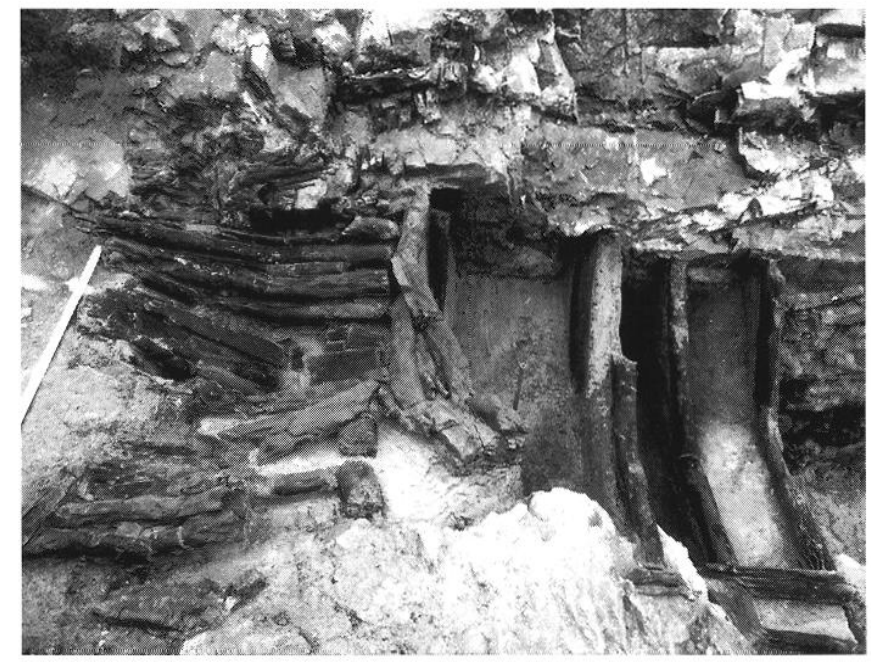

a

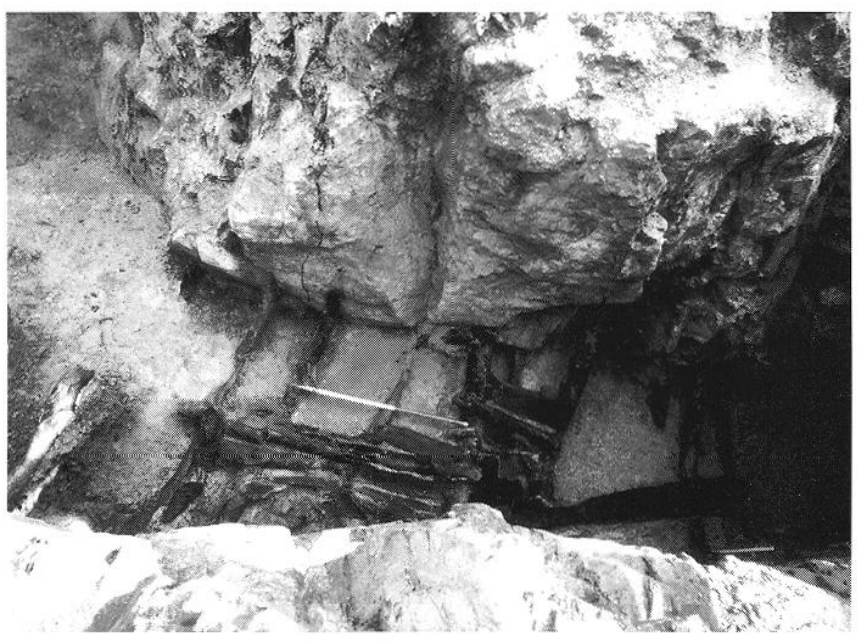

b

Fig. 66 - La Fagassière (Château-Chervix, Haute-Vienne), dépilage DEP3, plates-formes bâties en plancher: a, plate-forme supérieure; $b$, plate-forme inférieure.

le niveau du sol du fond de la fosse, situé à la cote $-8,50 \mathrm{~m}$ par rapport au rebord supérieur de l'excavation. Elle se trouvait en surplomb au-dessus du dépilage profond de plus de $5 \mathrm{~m}$ (fig. 66a).

Le second plancher occupait la partie centrale de l'ouvrage, à la cote - $12 \mathrm{~m}$ et à la profondeur d'environ $3 \mathrm{~m}$ à l'intérieur du chantier. Ce plancher était fait de cinq planches en chêne (largeur moyenne $0,30 \mathrm{~m}$, longueur entre 1,50 et $2,70 \mathrm{~m}$ ), posées sur sept étais transversaux et trois planches plus courtes (largeur moyenne $0,90 \mathrm{à} 1 \mathrm{~m}$ ) appuyées sur deux étais. Il couvrait en angle droit le côté ouest du dépilage sur $2,60 \mathrm{~m}^{2}$ (fig. 66b).
Ces deux planchers constituaient des plates-formes de travail où les mineurs pouvaient se tenir pour effectuer, en s'aidant d'une corde, la remontée vers la surface du minerai ou des déblais depuis le fond du dépilage en cours d'approfondissement. La plate-forme centrale servait de point de relais entre le fond et la surface. En effet, elle se trouvait, côté sud, à l'aplomb de la plateforme supérieure, ce qui permettait aux mineurs de se passer des charges (en sacs ou en paniers), d'un point de levage à l'autre. De même, ces plates-formes permettaient de stocker momentanément les pièces de bois nécessaires à l'étayage du chantier, au fur et à mesure de son approfondissement (fig. 67).

\section{PLATE-FORME EN REMBLAIS}

L'extrémité nord-est du dépilage était occupée par une troisième plate-forme. Elle était bâtie en remblais, adossés à une murette faite de blocs de quartz grossièrement appareillés sur plus de $2 \mathrm{~m}$ de hauteur. Cet espace, aux parois latérales maintenues par l'étayage complexe décrit supra (des planches de coffrage bloquées par des échelles faites d'étais assemblés à tenons et mortaises), était comblé par près de $7 \mathrm{~mm}^{3}$ de remblais $(1,80 \mathrm{~m}$ x 2,50 m, sur $3 \mathrm{~m}$ d'épaisseur) contre la paroi nord, qui était taillée en plan incliné, selon le pendage d'une ligne de faille. Sept niveaux de bois, disposés perpendiculairement à la murette, armaient le remblai. Cet aménagement constituait une plate-forme couvrant $4,50 \mathrm{~m}^{2}$ et surplombant le fond du dépilage côté nord (Cauuet, 1997a, p. 212-214).

De plus, une large planche $(0,30 \mathrm{~m} \times 1,80 \mathrm{~m})$ placée à $0,80 \mathrm{~m}$ en contrebas de la murette, et en appui sur deux étais transversaux, semblait marquer l'emplacement d'un poste de levage permettant de remonter et d'évacuer déblais et minerai (fig. 64).

Les trois plates-formes trouvées dans ce dépilage, associées au système d'étayage, révèlent une organisation de l'espace souterrain minier sur un plan vertical et horizontal. Le boisage disposé transversalement, outre sa fonction d'étayage, tenait lieu d'échelles aux mineurs pour leur progression verticale. Les plates-formes installées à différentes profondeurs, aux extrémités et au milieu de l'ouvrage, offraient des surfaces de relais pour décharger et stocker les produits à remonter ou les matériaux (boisages) à descendre (fig. 67). 


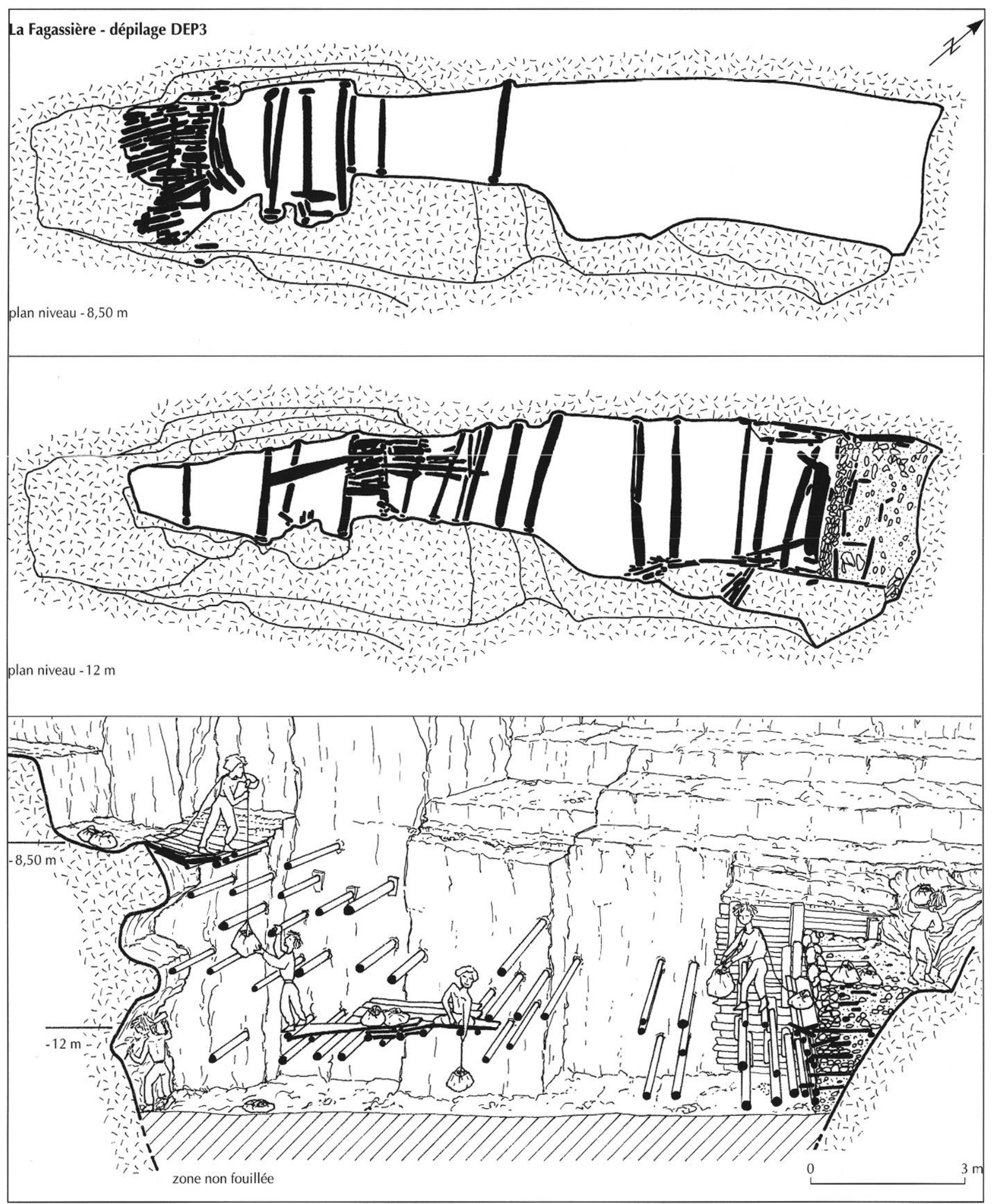

Fig. 67 - La Fagassière (Château-Chervix, Haute-Vienne) : plates-formes de travail en plancher et en remblais, installées à différents niveaux du dépilage IEP3; plans et restitution du dépilage vu en coupe. 


\section{VOLUMES DE BOIS MIS EN CEUVRE}

\section{EXEMPLE DE LA MINE DES FOUILLOUX}

L'ampleur de la fouille menée sur la mine des Fouilloux autorise à proposer des restitutions de volumes de bois mis en ouvre dans les dépilages de ce site. Les chantiers ouverts en enfilade, à partir du fond des grandes excavations, formaient un ensemble étiré sur près de $180 \mathrm{~m}$ de longueur. Les conditions de la fouille préventive menée en plusieurs phases, en 1988, puis en 1992 et 1994, n'ont pas permis d'étudier la totalité du fond des fosses. Côté sud, dans la partie centrale de la fosse F1, ainsi que côté nord, au niveau de F2 et de la plus grande partie de F4 (fig. 55), lors de la reprise d'exploitation du site par la Société des mines du Bourneix, la pelle mécanique a arraché de grandes quantités de boisages dans tous ces secteurs, sans que les chantiers d'où provenaient ces boisages aient pu être dénombrés.

Cependant, les dépilages étudiés ont révélé des types de boisages quasi identiques sur l'ensemble des ouvrages. De sorte que, par analogie, il est possible de restituer les ouvrages détruits avant étude et leurs boisages dans une vision globale du site. Il ressort de l'étude une modélisation des dépilages dont le système d'étayage peut être généralisé.

\section{ESSAI DE MODÉLISATION}

La modélisation choisie revient à subdiviser l'espace occupé par ces ouvrages dans la mine (des sortes de tranchées inclinées) en un nombre de chantiers aux proportions identiques. Ces derniers sont représentés régulièrement espacés par des piliers de roche laissée entre les chantiers pour maintenir en partie les terrains en place, le boisage assurant le reste de l'étayage à l'intérieur des ouvrages.

Compte tenu des observations recueillies dans la partie la mieux connue de la mine (côté sud), on peut faire le raisonnement suivant: si les dimensions données au dépilage sont de $1,50 \mathrm{~m}$ de large, $10 \mathrm{~m}$ de long et $10 \mathrm{~m}$ de profondeur, et si la distance couverte par les dépilages est de $180 \mathrm{~m}$, il faut compter 15 dépilages de $10 \mathrm{~m}$ de longueur chacun, séparés les uns des autres par des piliers de roche de $2 \mathrm{~m}$ de largeur, à savoir: $10 \mathrm{~m}$ x 15 dépilages $+2 \mathrm{~m} \times 14$ piliers $=178 \mathrm{~m}$ de longueur.

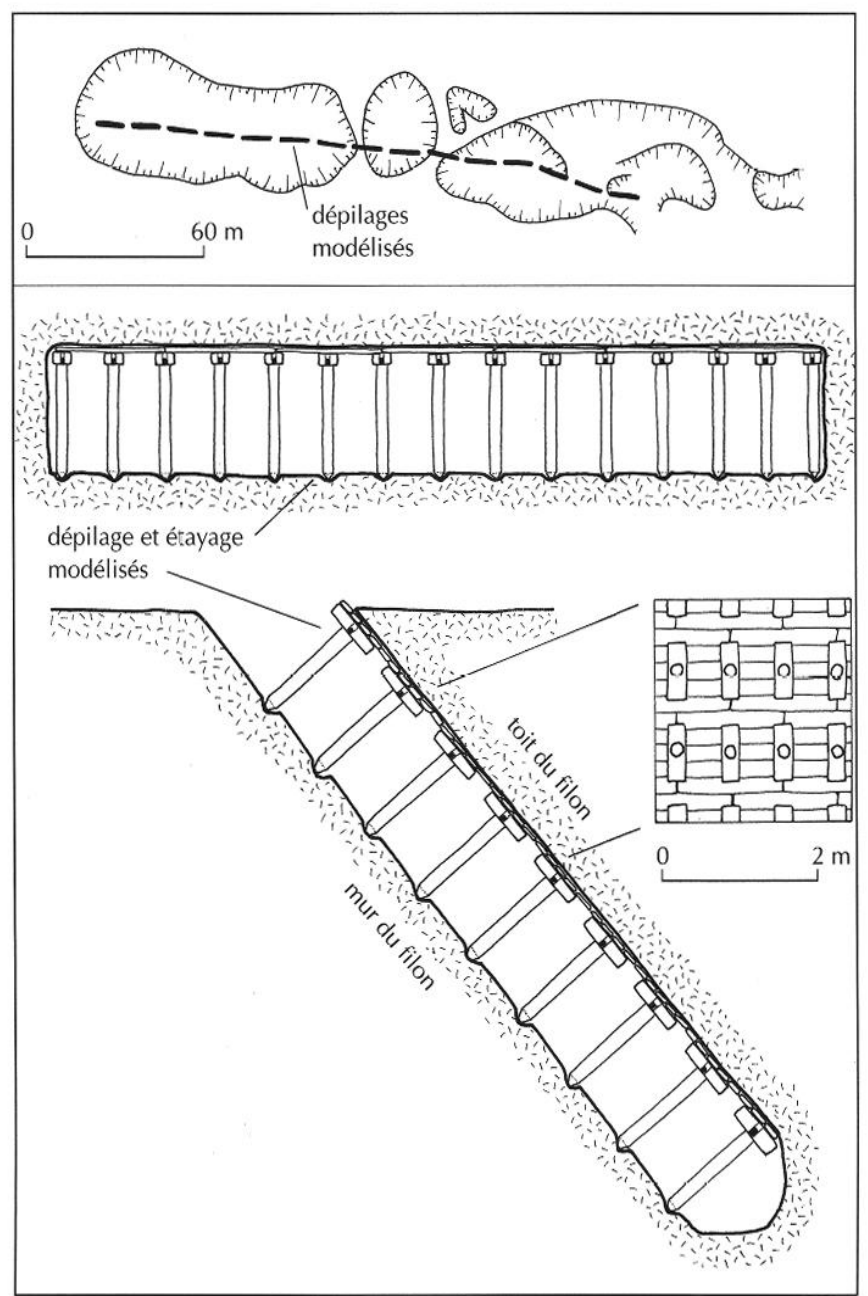

Fig. 68 - Les Fouilloux (Jumilhac-le-Grand, Dordogne) : modélisation des dépilages et des boisages.

L'étayage restitué dans chaque dépilage (selon le système observé in situ) comprend des pièces maitresses (poussard + montant), assemblées en " $T$ » par tenons et mortaises, et un bardage de palplanches qui couvre la paroi du côté du toit. Sachant que l'on trouve 1 assemblage en « $T$ » tous les $0,50 \mathrm{~m}$ dans la longueur, 1 rang de pièces assemblées tous les $0,75 \mathrm{~m}$ dans la profondeur (espaces observés in situ), et compte tenu des dimensions normalisées des trois types de pièces de bois considérées dans l'étayage (tabl. III), il en résulte que chaque dépilage comprend 15 assemblages en « $\mathrm{T}$ » dans la longueur et 9 assemblages dans la profondeur, ce qui donne: $15 \times 9=135$ jeux de pièces de bois assemblées en « $\mathrm{T}$ », correspondant à 135 poussards et 135 montants; donc un total de 270 étais par dépilage (fig. 68). 
Tabl. III - Dimensions normalisées et volumes des trois types de pièces de bois mises en place dans le dépilage des Fouilloux.

\begin{tabular}{|c|c|c|}
\hline Type de pièce & Dimensions & $\begin{array}{c}\text { Volume } \\
\text { (avant travail des pièces) }\end{array}$ \\
\hline $\begin{array}{l}\text { poussard diagonal } \\
\text { (tronc aux extrémités } \\
\text { travaillées en tenon et } \\
\text { en tronc de cône) }\end{array}$ & $\begin{array}{l}\text { diamètre : 0,15 m } \\
\text { longueur : } 1,50 \mathrm{~m}\end{array}$ & $\begin{array}{c}\left(r^{2}=0,0056\right) \\
(0,0056 \times 1,50 \times 3,14) \\
\mathbf{0 , 0 2 6} \mathbf{~ m}^{\mathbf{3}}\end{array}$ \\
\hline $\begin{array}{l}\text { montant oblique } \\
\text { ( } 1 / 2 \text { tronc débité sur dosses } \\
\text { et mortaisé en son centre) }\end{array}$ & $\begin{array}{l}\text { largeur : 0,25 m } \\
\text { longueur : 0,70 m } \\
\text { épaisseur : 0,15 m }\end{array}$ & $\begin{array}{c}(0,25 \times 0,70 \times 0,15) \\
\mathbf{0 , 0 2 6} \mathbf{~ m}^{\mathbf{3}}\end{array}$ \\
\hline planche & $\begin{array}{l}\text { largeur : 0,20 m } \\
\text { longueur : } 1,43 \mathrm{~m} \\
\text { ép. moyenne : } 0,05 \mathrm{~m}\end{array}$ & $\begin{array}{c}(0,20 \times 1,50 \times 0,05) \\
\mathbf{0 , 0 1 4} \mathbf{~ m}^{\mathbf{3}}\end{array}$ \\
\hline
\end{tabular}

Tabl. IV - Quantilés et volumes des pièces d'étayage utilisées aux Fouilloux.

\begin{tabular}{|c|c|c|c|}
\hline Type de pièce & $\begin{array}{c}\text { Nombre de pièces } \\
\text { par dépilage }\end{array}$ & $\begin{array}{l}\text { Nombre de pièces } \\
\text { pour } 15 \text { dépilages }\end{array}$ & Volume des pièces \\
\hline poussard & 135 & 2025 & $\begin{array}{c}\left(0,026 \mathrm{~m}^{3} \times 2025\right) \\
52,65 \mathrm{~m}^{3}\end{array}$ \\
\hline montant & 135 & 2025 & $\begin{array}{c}\left(0,026 \mathrm{~m}^{3} \times 2025\right) \\
52,65 \mathrm{~m}^{3}\end{array}$ \\
\hline planche & 280 & 4200 & 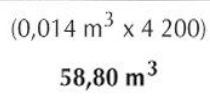 \\
\hline Totaux & 550 & 8250 & $164,10 \mathrm{~m}^{3}$ \\
\hline
\end{tabular}

Le bardage de palplanches fait d'épaisses planches, plaquées contre la paroi par les couples d'étais et disposées jointives les unes en dessous des autres, constitue un ensemble qui comprend par dépilage 7 planches dans la longueur et 40 planches dans la profondeur, soit un total de $7 \times 40=280$ planches.

On constate que chaque dépilage aura nécessité la mise en place de 550 pièces de bois, dont 270 étais et 280 planches (tabl. IV). À l'échelle du site et de 15 chantiers distincts, cela représente 4050 étais et 4200 planches, soit un total de 8250 pièces de bois. Par ailleurs, on n'oubliera pas de mentionner le travail nécessaire à la préparation des assemblages en « $T$ » qui correspond au percement de 2025 mortaises, à la taille de 2025 tenons et à l'épointement de 2025 étais pour faciliter leur logement dans les encoches creusées dans la paroi.

Selon la modélisation proposée, le volume global du bois mis en œuvre dans la mine des Fouilloux, à savoir près de $190 \mathrm{~m}^{3}$ (soit $164,10 \mathrm{~m}^{3}+24,61 \mathrm{~m}^{3}$ égal à $15 \%$ de chute), serait équivalent à un cube de bois de $5,80 \mathrm{~m}$ de côté. En outre, cette estimation ne tient pas compte des remplacements de pièces détériorées au cours de l'activité et attestés par la dendrochronologie (Szépertyski, 1999, p. 80).

Les trois types de pièces considérées donnent trois largeurs de tronc, à savoir:

- un fût de chêne presque centenaire, d'un diamètre de $45 \mathrm{~cm}$, pour les planches;

- un fût de chêne de 30 ans d'âge environ, d'un diamètre de $25 \mathrm{~cm}$, pour les montants;

- un fût de hêtre ou de chêne de 18 à 20 ans d'âge en moyenne, d'un diamètre de $15 \mathrm{~cm}$, pour les poussards.

D'un fût de chêne de $45 \mathrm{~cm}$ de largeur, on peut tirer 24 planches débitées en rayons (fig. $59, \mathrm{n}^{\circ} 3$ ). Compte tenu des largeurs $(20 \mathrm{~cm}$ ) et des longueurs (environ $150 \mathrm{~cm}$ ) des planches, on ne doit pouvoir tirer que deux fois 24 planches dans la hauteur d'un fût de cette largeur. Sachant qu'il faut débiter 4200 planches, cela revient à abattre 88 chênes centenaires ( 4200 : [24 x 2] $=87,5$ arbres). Un tel nombre d'arbres, de $15 \mathrm{~m}$ d'envergure en moyenne, peut correspondre à 1 ha de vieilles futaies de chênes.

D'un tronc de chêne de $25 \mathrm{~cm}$ de largeur, on tire deux montants débités sur dosses (fig. $59, \mathrm{n}^{\circ} 1$ ). Compte tenu des largeurs $(25 \mathrm{~cm})$ et des longueurs $(70 \mathrm{~cm})$ des montants, on ne peut tirer, au maximum, que cinq fois 2 montants dans la hauteur d'un tronc de cette largeur. Sachant qu'il faut débiter 2025 montants, cela revient à abattre 203 arbres ([2025:2]:5 = 202,5 arbres). Un tel nombre d'arbres, de $8 \mathrm{~m}$ d'envergure en moyenne, peut correspondre à 1,5 ha de chênes d'une trentaine d'années.

Enfin, compte tenu des largeurs $(15 \mathrm{~cm})$ et des longueurs $(150 \mathrm{~cm})$ des poussards, dans la hauteur d'un chêne ou d'un hêtre jeune (18 à 20 ans d'âge moyen), on peut tirer environ trois troncs (fig. 59). Sachant qu'il faut débiter 2025 poussards, cela revient à abattre 675 arbres (2025: 3) d'un diamètre moyen de $15 \mathrm{~cm}$. Un tel nombre d'arbres, de $7 \mathrm{~m}$ d'envergure environ, peut correspondre à 3,5 ha de chênes ou de hêtres de 18 à 20 ans d'âge.

L'ensemble de ces espaces boisés correspond à 6 ha au moins plantés, pour moitié en chênes, pour moitié en hêtres. Donc, la seule mine des Fouilloux a vraisemblablement conduit à l'abattage de 6 ha de forêt pour le soutènement de ces chantiers souterrains, aux II ${ }^{\mathrm{c}}$ et $\mathrm{I}^{\mathrm{er}} \mathrm{s}$. avant J.-C. Cela donne une idée de la pression exercée 
par les mines d'or du Limousin sur leur environnement naturel et que l'on doit prendre en compte dans l'analyse de cette activité économique en Gaule.

$*$

* *

Les assemblages à tenons et mortaises, l'étayage dense et continu des ouvrages révèlent une bonne connaissance du soutènement adapté à l'instabilité des terrains traversés, notamment en zone de surplomb et de faille. L'analyse des bois a montré que les techniques de débitage ont privilégié la refente. Elle demandait peu d'outillage, mais certainement de la force.

Les boiseurs ont choisi pour les étais transversaux des arbres jeunes et des essences disponibles dans le voisinage immédiat de la mine, et ceci, même si les essences utilisées, comme le hêtre et le bouleau, ne sont pas forcément adaptées à la mine humide. Pour les pièces travaillées, comme les montants et les planches, ils ont coupé des chênes anciens, à large fût, donnant de belles pièces utilisables et réutilisables sur le long terme.

Les chutes de planches ont servi de coins de calage. Les petites chutes de bois, les feuillages des branchages, les mousses et les fougc̀rcs des bois avoisinant les sites ont été employés comme bourrage derrière les boisages. En bref, rien ne s'est perdu, on a réutilisé les bois et fait durer le matériau.

Les essences n'ont sans doute pas été systématiquement sélectionnées. Les boiseurs se sont adaptés à leur environnement immédiat, utilisant la hêtraie et les bouleaux qui recolonisaient les espaces éclaircis par le défrichement des futaies anciennes de chêne. En effet, les larges planches et les montants souvent retrouvés en remploi dans les étayages correspondent à des arbres centenaires, jusqu'à 240 ans d'âge, abattus pour certains au III ${ }^{e}$ s. avant J.-C. (Szépertyski, 1999).

Outre la mise en place d'un étayage solide et ingénieux, on aura noté que ces mineurs gaulois aménageaient dans leurs chantiers souterrains différentes plates-formes de travail qui facilitaient la circulation et le stockage des produits de la mine. Le boisage, qu'il ait été simple ou complexe, était mis en place au rythme de l'approfondissement de l'exploitation. Son analyse dendrochronologique permet donc, par la datation des bois utilisés, de suivre la progression de la mine dans le temps, tout en permettant de conforter les observations de la fouille sur les travaux de réfection des boisages.

L'analyse palynologique vient compléter la restitution du couvert forestier qui entourait les mines, tout en révélant la présence naturelle de bois d'appoint, comme le hêtre et le bouleau. Ces essences ont sans doute été choisies parce qu'elles se trouvaient à portée de main, alors que d'autres, présentes également, comme l'aulne, n'ont pas été utilisées. Peutêtre a-t-on recherché plutôt la solidité et la durabilité des bois que leur bonne tenue en milieu humide. En effet, l'aulne résiste mieux à l'humidité, mais il a moins de dureté et de durabilité.

Enfin, on remarquera les grandes quantités de bois mises en œuvre dans ces mines, à l'origine vraisemblablement d'un défrichement important autour des sites, qui a dû provoquer un éclaircissement notable des chênaies anciennes. Ce travail particulier devait occuper des équipes de bûcherons et de charpentiers (ou boiseurs), à temps plein, pour fournir et équiper la mine en boisages. L'estimation de la main-d'œuvre spécialisée travaillant dans ces mines est une autre question, fort intéressante, sur laquelle nous travaillons, et qui sera traitée dans un prochain article. 